\title{
Age of Information in a Cooperative Slotted Aloha Network: Marginal and Joint Distributions
}

This paper was downloaded from TechRxiv (https://www.techrxiv.org).

\section{LICENSE}

CC BY 4.0

SUBMISSION DATE / POSTED DATE

25-08-2021 / 26-08-2021

\section{CITATION}

Vaezi, Kaveh; Akar, Nail; Karasan, Ezhan (2021): Age of Information in a Cooperative Slotted Aloha Network: Marginal and Joint Distributions. TechRxiv. Preprint. https://doi.org/10.36227/techrxiv.16437330.v1

$\mathrm{DOI}$ 


\title{
Age of Information in a Cooperative Slotted Aloha Network: Marginal and Joint Distributions
}

\author{
Kaveh Vaezi, Nail Akar, Member, IEEE, and Ezhan Karasan, Member, IEEE
}

\begin{abstract}
In this paper, we investigate a slotted Aloha cooperative network where a source node and a relay node send status updates of two underlying stochastic processes to a common destination. Additionally, the relay node cooperates with the source by accepting its packets for further retransmissions, where the cooperation policy comprises acceptance and relaying probabilistic policies. Exact marginal steady state distributions of the source and relay Age of Information (AoI) and Peak AoI (PAoI) sequences are obtained using Quasi-Birth-Death (QBD) Markov chain models. Extending this approach, we also obtain the joint distribution of the source and relay AoI sequences out of which one can obtain the steady state distribution of the Squared Difference of the two AoI sequences (SDAoI), which finds applications in network scenarios where not only the timeliness of status updates of each process is desired but also their simultaneity is of crucial importance. In this regard, we numerically obtain the optimal cooperation policy in order to minimize the expected value of SDAoI subject to a constraint on the average PAoI of the relay. Finally, our proposed analytical approach is verified by simulations and the performance of the optimal policy is discussed based on the numerical results.
\end{abstract}

Index Terms-Age of information, Cooperative networks, Peak age of information, Relays, Slotted Aloha

\section{INTRODUCTION}

In wireless status update systems, individual nodes send wirelessly the status of certain underlying stochastic processes towards a common destination (or monitor) in the form of information packets. In such systems, the timeliness of the status updates at the destination is of crucial importance. For the purpose of quantifying the timeliness, for a given node, the Age of Information (AoI) sequence is defined as the elapsed time since the generation instance of the last information packet that has been delivered to the destination [1]. As a result, this AoI sequence is a cyclic sequence which increases steadily in a cycle but is subject to abrupt downward jumps at the end of a cycle, the latter occurring at information packet reception instances. The Peak AoI (PAoI) sequence is obtained by concatenating the per-cycle peak values of the AoI sequence [2]. The expected values of the stationary AoI and PAoI sequences in discrete-time and of the AoI and PAoI processes in continuous-time have been extensively studied in the literature for a wide variety of communication scenarios whereas to a lesser extent, their higher order moments or distributions are sought; see the recent surveys on AoI [3], [4] and the references therein. On the other hand, cooperative scenarios are of great interest in a wide range of scenarios including cellular wireless networks, ad-hoc wireless

The authors are with the Department of Electrical and Electronics Engineering, Bilkent University, Ankara, Turkey (e-mail: $\{$ kaveh,akar,ezhan\}@ee.bilkent.edu.tr). networks, next generation communication networks, etc. due to their ability to improve the network performance in terms of throughput and delay. However, AoI-related metrics have not been extensively studied in cooperative status update system scenarios where certain nodes cooperate, in some means, to transmit their time-sensitive packets to the destination.

In this paper, we investigate AoI and PAoI in a cooperative slotted Aloha network in which the source (S) and the relay $(\mathrm{R})$ nodes send status updates of the source and relay processes, respectively, to a common destination (D) in a timely manner; see Fig. 1. Moreover, the relay node attempts to reduce the AoI of the source node by devoting its own transmission resources for relaying source packets held at a single-packet queue (Q) at the expense of increasing the AoI of the relay node itself. In the studied setting, the relay node controls the level of cooperation by adopting two probabilistic policies namely the admission and relaying policies, the combination of these two policies being referred to as the cooperation policy. This basic cooperative scheme has been considered in various existing works [5]-[8].

Our first goal here is to obtain the distribution of AoI and PAoI sequences associated with the source and relay nodes. The difference between the source and relay AoI sequences is also of crucial importance in some applications. For example, consider a simple sensor network comprising two sensors, one of which reports the wind speed in the environment under surveillance (e.g., a forest susceptible to wildfire) and the other one informs about the wind direction. The wind speed and direction are both decisive parameters to prevent or control a wild fire, which should be known in a timely manner and also simultaneously. As another example, consider a self-driving vehicle moving in between two other vehicles at its back and front. The self-driving system should be aware of the speed of the adjacent vehicles to prevent a crash. Although the timeliness of speed information of each vehicle is important, the simultaneity of the collected information of the two vehicles is also vital. Motivated by these observations, we also the steady state distribution of the SDAoI (Squared Difference AoI) sequence which is the squared difference between the source and relay AoI sequences. We note that the distribution of the SDAoI sequence can directly be obtained from the joint distribution of the source and relay AoI sequences, obtaining the latter being the second goal of this paper.

The main contributions of this paper are as follows:

- Exact steady state distributions of AoI and PAoI sequences of the source and relay processes as well as the exact steady state joint distribution of the two AoI sequences are obtained using a computationally stable and efficient numerical algorithm. To the best of our knowledge, the 


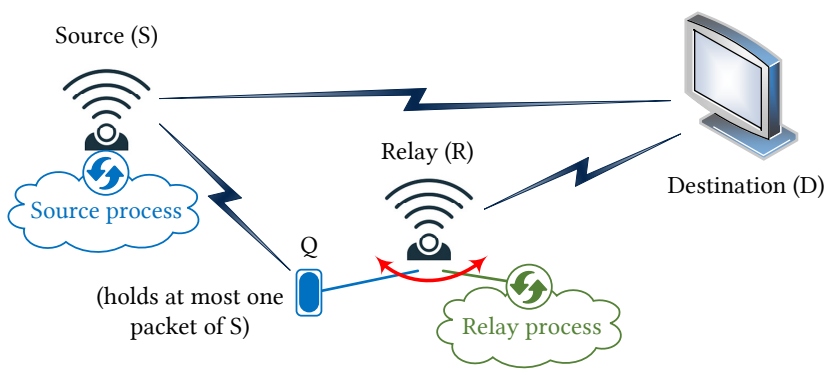

Fig. 1: The studied two-node cooperative slotted Aloha-based scenario.

current work is the first attempt at exactly obtaining the joint distribution of AoI sequences of multiple processes, in relaying context.

- Thanks to the computational efficiency of the proposed algorithm, one can obtain an optimum cooperation policy using brute-force optimization on the admission and relaying parameters of the underlying cooperation policy in order to minimize a given cost. In the numerical examples, we minimize the expected value of the steady state SDAoI subject to constrained average PAoI of the relay node.

The rest of the paper is organized as follows: In Section II, we present related work. Section III details the system model. Marginal steady state distributions of AoI and PAoI sequences are derived numerically in Section IV while their joint steady state distribution is obtained in Section V. The specific optimization problem we address is described in Section VI. In Section VII, we provide our numerical results. Finally, Section VIII concludes the paper.

\section{RELATED WORK}

The introduction of the AoI concept in [1], [9] has recently caused a surge of interest in AoI-related research. In the earlier works, AoI is basically studied from a continuoustime queueing systems viewpoint. In [1], the average AoI was obtained for $\mathrm{M} / \mathrm{M} / 1, \mathrm{M} / \mathrm{D} / 1$ and $\mathrm{D} / \mathrm{M} / 1$ queues with First-Come-First-Served (FCFS) discipline and optimal arrival rates are obtained so as to minimize the average AoI. The probability distribution function of AoI and PAoI in FCFS $\mathrm{M} / \mathrm{M} / 1 / 1$ and $\mathrm{M} / \mathrm{M} / 1 / 2$ queues as well as the non-preemptive Last-Come-First-Served (LCFS) M/M/1/2* queue are studied in [10]. Recently, the authors of [11] derived a general formula for the stationary distribution of the AoI, which holds for a wide class of single-source information update systems. Also, the exact distributions of AoI and PAoI are obtained numerically for a bufferless $\mathrm{PH} / \mathrm{PH} / 1 / 1$ queue with probabilistic preemption as well as a single buffer $\mathrm{M} / \mathrm{PH} / 1 / 2$ queue with probabilistic replacement of the waiting packet [12]. Multi-source systems have also attracted the attention of researchers. In [13], the authors study a multi-source $\mathrm{M} / \mathrm{M} / 1$ queue with FCFS, preemptive bufferless, and non-preemptive single buffer with replacement queueing disciplines, using Stochastic Hybrid Systems (SHS) and derive the mean AoI for each source. Moreover, the authors find the region of feasible average status ages for multiple updating sources and also characterize how a service facility can be shared among multiple sources. The reference [14] obtains the average PAoI for $\mathrm{M} / \mathrm{G} / 1$ and $\mathrm{M} / \mathrm{G} / 1 / 1$ systems with heterogeneous service time requirements. A two-source $\mathrm{M} / \mathrm{M} / 1 / 2$ queueing system is studied using SHS techniques in [15] where a packet waiting in the queue is replaced by a newly arriving packet from the same source.

A number of research studies focus on discrete-time AoI queueing models. The authors of [16] study the FCFS-type Ber/G/1 queue and derive explicit expressions for average AoI and PAoI and also mean AoI expressions for the discretetime LCFS queue. In the recent work [17], the authors investigate a single source discrete-time system for FCFS, preemptive LCFS, and bufferless (with packet dropping) queueing schemes and they derive closed-form expressions for the generating functions and the stationary distributions of the AoI and the PAoI. In another recent work [18], the authors study a discrete time multi-source system using the instrument of QBD Markov chains and obtain the exact distribution of per-source AoI and PAoI for systems with non-preemptive bufferless, preemptive bufferless and non-preemptive single buffer with replacement queueing disciplines.

More recently, a shift of interest to multi-hop scenarios can be witnessed. The reference [19] investigates a general multi-hop network where packets of a source disperse through the network from an entry point. The authors show that if the transmission time of packets is exponentially distributed, among all possible scheduling policies of the nodes, LastGenerated-First-Served preemptive policy minimizes the average age of the stream. The authors of [20] consider a time-slotted network scenario where a source node transmits the status updates to a destination with the help of a relay node while the direct link also exists and the transmissions of source and relay nodes are scheduled. Two age-oriented relaying protocols are devised in this work to reduce the AoI at destination, and the closed-form average AoI is derived for both protocols. The reference [21] investigates a two-hop continuous-time system where status updates are captured by both nodes, however, higher priority is considered for the packets that travel through the two-hop link to destination. Exact distributions of AoI and PAoI are derived for the nonpriority packets while tight bounds are found for the priority flow. In this paper, we study a two-hop network in discretetime for which transmissions of source and relay nodes use random access which is a different scenario than the ones that are mentioned above. AoI has also been studied for noncooperative random access networks as well. As an example, [22] investigates a scenario where sources send their status updates to a destination using a variation of slotted Aloha and obtains the average AoI while comparing the proposed random access method with conventional slotted Aloha and round robin protocols.

Investigating AoI in random access networks has attracted the attention of several researchers. To name a few, the author of [23] studies the impact of Irregular Repetition Slotted Aloha (IRSA) on AoI. IRSA is a modern random access protocol which have recently emerged as promising solutions to support massive machine-type communications. In this work, the steady state distribution of AoI and its expected value are derived in closed-form. [24] proposes decentralized ageefficient transmission policies for random access channels with 
M transmitters and shows that the slotted Aloha-type algorithms are asymptotically age-optimal only when the arrival rates are less than a specified threshold. Authors in [25] present a steady-state analysis of threshold-Aloha, which is a distributed age-aware modification of slotted Aloha. In thresholdAloha, each node suspends its transmission until a certain age threshold is reached and then it attempts to transmit with a fixed probability. Also, the authors of [26] propose an ageoriented random access protocol for massive IoT networks, whereas [27] studies AoI in random access networks with stochastic arrivals. AoI has also been studied in Carrier-Sense Multiple Access (CSMA) environments [28], [29]. In [30], a CSMA-based random access framework is proposed, in which the transmission probabilities on nodes in the contention period is determined by their respective inner-states.

One essential instrument that we use in this paper is the Quasi-birth-Death (QBD) Markov chain model, which is briefly described next based on [31]. The infinite QBDtype discrete-time Markov chain, denoted by $\Xi$, is a twodimensional Markov chain $X_{\ell}=\left(L_{\ell}, P_{\ell}\right)$ where $L_{\ell}, 0 \leqslant L_{\ell} \leqslant$ $\infty$ represents the level sequence of the QBD and the finitedimensional $P_{\ell}$ stands for the phase sequence, and $\Xi$ has an irreducible probability transition matrix $P_{\Xi}$ of the following canonical block tridiagonal form:

$$
P_{\Xi}=\left[\begin{array}{ccccc}
A_{00} & A_{01} & & & \\
A_{10} & C & D & & \\
& B & C & D & \\
& & \ddots & \ddots & \ddots
\end{array}\right],
$$

with $A_{00}, A_{01}$, and $A_{10}$ being non-negative matrices of sizes $i \times i, i \times j, j \times i$, respectively, and the matrices $B, C$, and $D$ being of size $j \times j$. For a positive recurrent QBD, the stationary probability vector $\boldsymbol{\pi}=\left[\boldsymbol{\pi}_{0}, \boldsymbol{\pi}_{1}, \ldots\right]$ of $\Xi$ exists where $\boldsymbol{\pi}_{k}$ is the solution vector for level $k$ and is of size $1 \times i$ for $k=0$ and $1 \times j$ for $k \geqslant 1$, and is the unique solution to the equations

$$
\boldsymbol{\pi}=\boldsymbol{\pi} P_{\Xi}, \quad \boldsymbol{\pi}_{0} \mathbf{1}_{i}+\sum_{k=1}^{\infty} \boldsymbol{\pi}_{k} \mathbf{1}_{j}=1,
$$

where $\mathbf{1}_{l}$ denotes a column vector of ones of size $l$. The stationary solution at level $k(k \geqslant 2)$ is in form of

$$
\boldsymbol{\pi}_{k}=\boldsymbol{\pi}_{1} \boldsymbol{R}^{k-1}, \quad k \geqslant 2,
$$

where the rate matrix $\boldsymbol{R}$ is the solution of the quadratic matrix equation $\boldsymbol{R}=B \boldsymbol{R}^{2}+C \boldsymbol{R}+D$, and can be obtained by computationally efficient algorithms with computational complexity $\mathcal{O}\left(j^{3}\right)$ such as the invariant subspace approach [32]. However, for the introduced QBDs in this paper, $C$ is all zeros and $B$ and $D$ are in the form

$$
B=\left[\begin{array}{cc}
B_{0} & B_{1} \\
O_{(j-l) \times l} & O_{l \times l}
\end{array}\right], D=\left[\begin{array}{cc}
O_{(j-l) \times(j-l)} & O_{l \times(j-l)} \\
O_{(j-l) \times l} & I_{l}
\end{array}\right],
$$

where $I_{l}$ indicates the identity matrix of size $l$ and $O$ is the all-zeros matrix of the specified size. In this case. the matrix $\boldsymbol{R}$ defined as

$$
\boldsymbol{R}=\left[\begin{array}{cc}
B_{0} & \left(I_{(j-l) \times(j-l)}-B_{0}\right)^{-1} B_{1} \\
O_{(j-l) \times l} & O_{l \times l}
\end{array}\right],
$$

can easily be shown to satisfy the aforementioned quadratic equation. Once $\boldsymbol{R}$ is computed, the vectors $\pi_{0}$ and $\pi_{1}$ can be obtained by solving the following linear matrix equations

$$
\begin{aligned}
& {\left[\boldsymbol{\pi}_{0}, \boldsymbol{\pi}_{1}\right]\left[\begin{array}{cc}
A_{00}-I_{i} & A_{01} \\
A_{10} & \boldsymbol{R} B-I_{j}
\end{array}\right]=\mathbf{0},} \\
& \boldsymbol{\pi}_{0} \mathbf{1}_{i}+\boldsymbol{\pi}_{1}\left(I_{j}-\boldsymbol{R}\right)^{-1} \mathbf{1}_{j}=1 .
\end{aligned}
$$

\section{SySTEM MODEL}

In this paper, we investigate a slotted Aloha-based cooperative wireless network where two nodes, namely the source node $\mathrm{S}$ and the relay node $\mathrm{R}$, send status update packets for two underlying stochastic processes, namely the source and relay processes, to a common destination $\mathrm{D}$. Moreover, $\mathrm{R}$ holds a so-called relay quеие $\mathrm{Q}$ to buffer the unsuccessfully transmitted packets of $\mathrm{S}$ for further re-transmissions. We assume that $\mathrm{Q}$ is a single buffer queue holding the last accepted packet received from S. Fig. 1 depicts the described network. Due to the random access mechanism, collision (simultaneous transmissions of more than one node) may happen in which case D is not able to decode any of the transmitted packets. The physical layer, i.e., channel fading characteristics or applied diversity and coding methods, is modeled by the success probability $\phi_{u, v}$ for transmission in the link $u$ - $v$, i.e., from node $u$ to node $v$, where $u \in\{\mathrm{S}, \mathrm{R}\}$ and $v \in\{\mathrm{R}, \mathrm{D}\}$. Each status update is generated at the onset of the time slot it is transmitted. Thus, status updates do not wait at nodes before they are transmitted.

At the beginning of each time slot, if $\mathrm{S}$ decides to transmit in that time slot with transmission probability $p_{\mathrm{S}}$, a status update of the source process is generated and sent. The transmitted packet is successfully delivered to D unless it is erased either due to channel errors (with erasure probability $1-\phi_{\mathrm{S}, \mathrm{D}}$ ) or due to collision. Upon successful transmission, an ACK message from $\mathrm{D}$ informs $\mathrm{R}$ of the successful transmission to drop the potential existing packet in Q. Note that, since the successfully transmitted packet via the direct link S-D is fresher than the waiting packet in $\mathrm{Q}$, there is no point to hold the packet in $\mathrm{Q}$ any more, thus $\mathrm{R}$ should drop it if it hears an ACK from D. However, in case of unsuccessful transmission in the direct link, R may also be able to decode the packet with probability $\phi_{\mathrm{S}, \mathrm{R}}$ provided that there is no collision. We assume that ACK messages are immediately heard by both source and relay nodes with no errors. Herein this paper, we assume that $\mathrm{R}$ adopts an admission controlled replacement policy at $\mathrm{R}$, that is once a packet of $\mathrm{S}$ is received at $\mathrm{R}$, it replaces the existing staler packet in $\mathrm{Q}$ provided that there is any, however, if $\mathrm{Q}$ is empty, then the new packet is accepted with the so-called acceptance probability $\alpha$. If $\mathrm{R}$ decides to send a packet with transmission probability $p_{\mathrm{R}}$ at the onset of a time slot, it should decide whether to transmit a newly generated status update of the relay process (its own packet) or the existing packet in $\mathrm{Q}$ (if any). Once $\mathrm{Q}$ is empty, $\mathrm{R}$ sends its own packet. Otherwise, $\mathrm{R}$ transmits the waiting packet in $\mathrm{Q}$ with the so-called relay probability $\beta$, and its own packet with probability $1-\beta$. Either way, the transmitted packet is received successfully at $D$ with the success probability $\phi_{\mathrm{R}, \mathrm{D}}$ unless a collision occurs. If the relayed packet of $\mathrm{S}$ is received successfully at $\mathrm{D}$, an ACK 
TABLE I: Possible events in the network and their probabilities

\begin{tabular}{|l|l|}
\hline Event & Probability \\
\hline succ. $^{\text {a from } \mathrm{S} \text { to D }}$ & $r_{\mathrm{S}, \mathrm{D}} \triangleq p_{\mathrm{S}}\left(1-p_{\mathrm{R}}\right) \phi_{\mathrm{S}, \mathrm{D}}$ \\
\hline succ. from S to R (if Q is full) & $r_{\mathrm{S}, \mathrm{R}} \triangleq p_{\mathrm{S}}\left(1-p_{\mathrm{R}}\right)\left(1-\phi_{\mathrm{S}, \mathrm{D}}\right) \phi_{\mathrm{S}, \mathrm{R}}$ \\
\hline succ. from S to R (if Q is empty) & $r_{\mathrm{S}, \mathrm{R}} \alpha$ \\
\hline $\begin{array}{l}\text { succ. from R (status update) to D } \\
\text { (if Q is empty) }\end{array}$ & $r_{\mathrm{R}, \mathrm{D}} \triangleq p_{\mathrm{R}}\left(1-p_{\mathrm{S}}\right) \phi_{\mathrm{R}, \mathrm{D}}$ \\
\hline $\begin{array}{l}\text { succ. from R (status update) to D } \\
\text { (if Q is full) }\end{array}$ & $r_{\mathrm{R}, \mathrm{D}}(1-\beta)$ \\
\hline succ. from Q to D (if Q is full) & $r_{\mathrm{R}, \mathrm{D}} \beta$ \\
\hline $\begin{array}{l}\text { unsucc. } \text { (from S or R ) } \\
\text { (due to channel inefficiencies) }\end{array}$ & $r_{u} \triangleq p_{\mathrm{R}}\left(1-p_{\mathrm{S}}\right)\left(1-\phi_{\mathrm{R}, \mathrm{D}}\right)$ \\
\hline No transmission (idle time slot) & $r_{i} \triangleq\left(1-p_{\mathrm{S}}\left(1-p_{\mathrm{R}}\right)\left(1-\phi_{\mathrm{S}, \mathrm{D}}\right)\left(1-p_{\mathrm{R}}\right)\right.$ \\
\hline Collision & $r_{c} \triangleq p_{\mathrm{S}, \mathrm{R}} p_{\mathrm{R}}$ \\
\hline Collision or unsucc. or idle & $r_{0} \triangleq r_{c}+r_{u}+r_{i}$ \\
\hline a successful transmission & $\mathrm{b}$ unsuccessful transmission \\
\hline
\end{tabular}

from $\mathrm{D}$ informs $\mathrm{R}$ to drop that packet from $\mathrm{Q}$. According to the described scenario, Table I provides the probabilities of all possible events in the network. For reference, we call the choice of the pair $(\alpha, \beta)$ as the cooperation policy of $\mathrm{R}$.

Let the discrete-time discrete-valued random sequence $\Delta_{u}(\ell)$ denote the AoI governed by the status update packets transmitted by node $u(u \in\{\mathrm{S}, \mathrm{R}\})$, monitored by $\mathrm{D}$ at time slot $\ell$. By definition, $\Delta_{u}(\ell)=\ell-\delta_{u}(\ell)$ where $\delta_{u}(\ell)$ is the instance of generation of the most recent received packet of $u$ at D. More specifically, suppose that the $j^{\text {th }}$ successful packet of node $u$ is generated at time slot $\delta_{u}^{j}$ and received successfully at $\mathrm{D}$ at time slot $\theta_{u}^{j}$. Then, $\Delta_{u}(\ell)$ starts from $\Delta_{u}\left(\theta_{u}^{j}\right)=\theta_{u}^{j}-\delta_{u}^{j}$ and increases monotonically in time until the next successful packet of $u$ is delivered successfully to D, i.e., time slot $\theta_{u}^{j+1}$, when $\Delta_{u}(\ell)$ suddenly drops to $\theta_{u}^{j+1}-\delta_{u}^{j+1}$. At the instance just before the $(j+1)^{\text {th }}$ packet is received, age of the $j^{\text {th }}$ packet takes the maximum value, namely $\theta_{u}^{j+1}-\delta_{u}^{j}$, which is called the peak age of the $j^{\text {th }}$ packet. The sequence $\Lambda_{u}(\ell)$, known as the peak AoI of packets of node $u$, is the sequence obtained by concatenating these peak values. Note that, since each packet takes at least one time slot to be received successfully at $\mathrm{D}, \Delta_{u}(\ell)$ takes the minimum value 1 . Consider the $j^{\text {th }}$ packet of node $u$ which is aged at $\mathrm{D}$ from time slot $\theta_{u}^{j}$ to $\theta_{u}^{j+1}-1$. The sub-sequence of $\Delta_{u}(\ell)$ where $\ell \in\left[\theta_{u}^{j}, \theta_{u}^{j+1}-1\right]$ is referred to as the age cycle of successful packet $j$ of node $u$. Therefore, $\Delta_{u}(\ell)$ is simply composed of successive age cycles of successively received packets of node $u$ at D. Let us denote the steady state probability mass function (pmf) of $\Delta_{u}(\ell)$ and $\Lambda_{u}(\ell)$ by $p_{\Delta_{u}}(n)$ and $p_{\Lambda_{u}}(n)$, respectively. Then,

$$
\begin{aligned}
& p_{\Delta_{u}}(n)=\lim _{\ell \rightarrow \infty} \operatorname{Pr}\left(\Delta_{u}(\ell)=n\right), \quad n \geqslant 1, \\
& p_{\Lambda_{u}}(n)=\lim _{\ell \rightarrow \infty} \operatorname{Pr}\left(\Lambda_{u}(\ell)=n\right), \quad n \geqslant 1,
\end{aligned}
$$

where $\operatorname{Pr}(\cdot)$ denotes the probability of its argument. Also, denoting the joint age sequence $\left(\Delta_{\mathrm{S}}(\ell), \Delta_{\mathrm{R}}(\ell)\right)$ by $\Psi(\ell)$, the steady state joint pmf of the sequences $\Delta_{\mathrm{S}}(\ell)$ and $\Delta_{\mathrm{R}}(\ell)$, denoted by $p(n, m)(\forall n, m \geqslant 1)$, is written as

$$
p(n, m)=\lim _{\ell \rightarrow \infty} \operatorname{Pr}(\Psi(\ell)=(n, m)) .
$$

Furthermore, $\Gamma(\ell)$ is defined as the Squared Difference of the source and relay AoI sequences, called the SDAoI sequence, i.e., $\Gamma(\ell)=\left(\Delta_{S}(\ell)-\Delta_{R}(\ell)\right)^{2}$ with its steady state value denoted by the random variable $\Gamma$. Specifically, the Mean SDAoI (MSD) can be obtained from $p(n, m)$ as follows:

$$
\bar{\Gamma}=E[\Gamma]=\sum_{n \geqslant 1} \sum_{m \geqslant 1}(n-m)^{2} p(n, m),
$$

where $E[\cdot]$ denotes the expectation operator.

\section{Marginal PMFs of the AoI And PAoI Sequences}

Inspired by [18], in order to derive the pmfs $p_{u_{\Delta}}(n)$ and $p_{u_{\Lambda}}(n)(u \in\{\mathrm{S}, \mathrm{R}\})$, we model the journey of each packet (originated by node $u$ ) from the instance of its generation and then its successful transmission to $\mathrm{D}$ (the beginning of the age cycle of the packet), till the instant at which the next packet is received successfully at $\mathrm{D}$ (when the age cycle ends), by a QBD-type infinite Markov chain $X_{\ell}=\left(L_{\ell}, P_{\ell}\right), \ell=1,2, \ldots$, as described in Section II. While the phases of this QBD show the state of the system regarding the packet of interest (whose journey is being modeled, denoted by $\mathrm{P}_{0}$ ), levels, starting from level 1 , model the elapsed time since the generation of $\mathrm{P}_{0}$. More specifically, once $\mathrm{P}_{0}$ is generated and transmitted successfully to another node, i.e., D or Q (for the packets of S), its journey starts at level 1. Following that, the QBD's level increases by 1 after each time slot, until the next packet of node $u$ is received successfully at $\mathrm{D}$, that is when the age cycle of $\mathrm{P}_{0}$ ends and the QBD should be restarted from level 1 to account for the journey of another packet. To maintain the QBD form of the Markov chain, we use certain auxiliary phases for restarting the QBD from level 1. These phases are described where the QBD models are detailed.

\section{A. QBD Model to Obtain $p_{\Delta_{\mathrm{R}}}(n)$ and $p_{\Lambda_{\mathrm{R}}}(n)$}

Once a packet of $\mathrm{R}$ (e.g., $\mathrm{P}_{0}$ ) is generated at the beginning of a time slot and delivered to $\mathrm{D}$ at the end of that time slot, its journey in the proposed QBD (named $\Xi_{\mathrm{R}}$, depicted in Fig. 2) begins from level 1 at phase $\mathrm{E}$ or $\mathrm{F}$, depending on whether $\mathrm{Q}$ is empty or full at that time, respectively. Following that, till the next successful transmission from $\mathrm{R}$ to $\mathrm{D}$, the state of $\mathrm{Q}$ may change due to transmissions from $\mathrm{S}$ or $\mathrm{Q}$, hence the phase of $\Xi_{R}$ may change between $E$ or $F$ in successive levels. When the next packet of $\mathrm{R}$ is delivered to $\mathrm{D}$, the age cycle of $P_{0}$ ends and $\Xi_{R}$ should be restarted from level 1, to account for the age cycle of the next packet of R. To this end, the auxiliary phases $T_{e}$ and $T_{f}$ are introduced to go back (level by level) from phases $\mathrm{E}$ and F, respectively. Note that if the new packet arrives at D when Q is empty (or full), $\Xi_{R}$ should be restarted at phase E (F, resp.). Transition probabilities are detailed in Fig. 2. Recalling the definition of $\Delta_{\mathrm{R}}(n)$ and $\Lambda_{\mathrm{R}}(n)$, the phases $\mathrm{K}_{\mathrm{e}}$ and $\mathrm{K}_{\mathrm{f}}$ are introduced in $\Xi_{\mathrm{R}}$ to find $p_{\Lambda_{\mathrm{R}}}(n)$. In fact, these states indicate what is the maximum value of age of $\mathrm{P}_{0}$ just before resetting to 1 (due to the new packet arrival). Thus, $p_{\Lambda_{\mathrm{R}}}(n)$ is found by

$$
\begin{aligned}
p_{\Lambda_{\mathrm{R}}}(n) & =\lim _{\ell \rightarrow \infty} \operatorname{Pr}\left(L_{\ell}=n \mid P_{\ell} \in\left\{\mathrm{K}_{\mathrm{e}}, \mathrm{K}_{\mathrm{f}}\right\}\right), \\
& =\frac{\pi_{\mathrm{R}}\left(n, \mathrm{~K}_{\mathrm{e}}\right)+\pi_{\mathrm{R}}\left(n, \mathrm{~K}_{\mathrm{f}}\right)}{\sum_{n=1}^{\infty} \pi_{\mathrm{R}}\left(n, \mathrm{~K}_{\mathrm{e}}\right)+\pi_{\mathrm{R}}\left(n, \mathrm{~K}_{\mathrm{f}}\right)},
\end{aligned}
$$




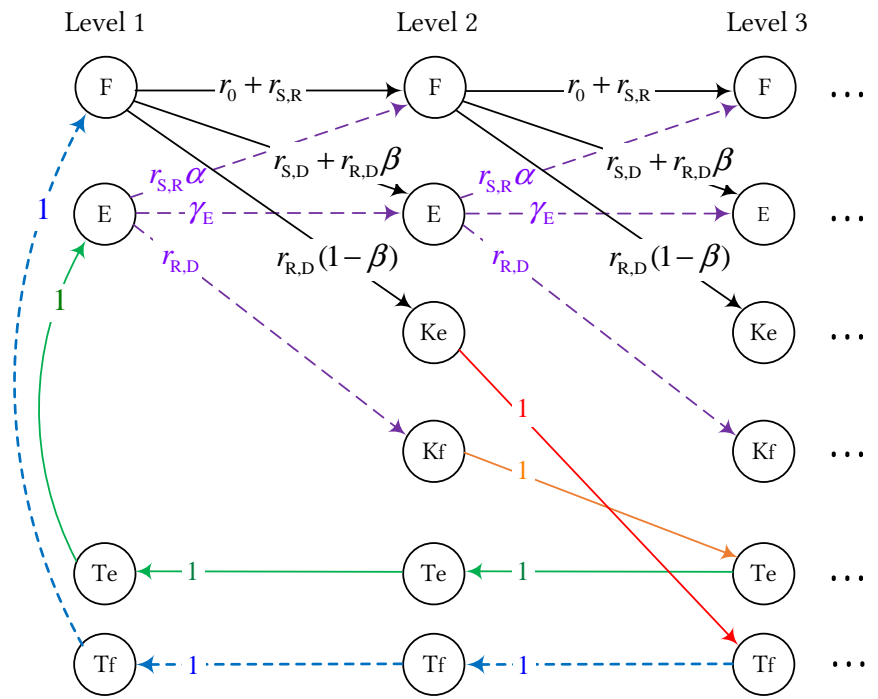

Fig. 2: The transition diagram of $\Xi_{\mathrm{R}}$, where $\gamma_{\mathrm{E}} \triangleq r_{0}+r_{\mathrm{S}, \mathrm{D}}+r_{\mathrm{S}, \mathrm{R}}(1-\alpha)$

where $\pi_{\mathrm{R}}(x)$ is the steady state probability of being at state $x$ in $\Xi_{R}$. Note that we introduce the auxiliary phases $T_{e}$ and $T_{f}$ only to restart $\Xi_{\mathrm{R}}$, and also the phases $\mathrm{K}_{\mathrm{e}}$ and $\mathrm{K}_{\mathrm{f}}$ to calculate $p_{\Lambda_{\mathrm{R}}}(n)$. Henceforth, $p_{\Delta_{\mathrm{R}}}(n)$ is written as

$$
\begin{aligned}
p_{\Delta_{\mathrm{R}}}(n) & =\lim _{\ell \rightarrow \infty} \operatorname{Pr}\left(L_{\ell}=n \mid P_{\ell} \in\{\mathrm{E}, \mathrm{F}\}\right), \\
& =\frac{\pi_{\mathrm{R}}(n, \mathrm{E})+\pi_{\mathrm{R}}(n, \mathrm{~F})}{\sum_{n=1}^{\infty} \pi_{\mathrm{R}}(n, \mathrm{E})+\pi_{\mathrm{R}}(n, \mathrm{~F})} .
\end{aligned}
$$

\section{B. QBD Model to Obtain $p_{\Delta_{\mathrm{S}}}(n)$ and $p_{\Lambda_{\mathrm{S}}}(n)$}

Investigating $\Delta_{S}(n)$ is different from $\Delta_{R}(n)$ as packets of $\mathrm{S}$ reach $\mathrm{D}$ via two links: the direct and relay links. Age cycle of the packets that traverse the direct link, starts from 1, while the packets that travel through the relay link wait at $\mathrm{Q}$ for some time slots before they are delivered to $\mathrm{D}$, provided that they are not replaced or dropped. Hence, the age cycle of the packet that passes the relay link, if not replaced or dropped, starts from a value larger than 1 (at least 2, due to 1 time slot for $\mathrm{S}$ to $\mathrm{R}$ and 1 time slot for $\mathrm{Q}$ to $\mathrm{D}$ transmissions). Therefore, in the proposed QBD (named $\Xi_{\mathrm{S}}$, refer to Fig. 3) that models the journey of packets of $\mathrm{S}$ (e.g., $\mathrm{P}_{0}$ ) in the network, the phase $\mathrm{W}$ is introduced to track the packets who travel through the relay link. Thus, once $P_{0}$ is accepted at $Q$, its journey in $\Xi_{S}$ starts from state $\mathrm{W}$ at level 1 . If the packet is replaced or dropped, $\Xi_{\mathrm{S}}$ is tracking a faulty packet, as age is not defined for a packet which is not received at D. In that case, $\Xi_{S}$ should start over at level 1 , tracking the newly received packet at $\mathrm{R}$ (that replaced $\mathrm{P}_{0}$ ) or $\mathrm{D}$ (which forced $\mathrm{R}$ to drop $\mathrm{P}_{0}$ ), which is done by the auxiliary phases $T_{p}$ and $T_{d}$, respectively. On the other hand, if $\mathrm{P}_{0}$ is not replaced or dropped, it is relayed to $\mathrm{D}$ by $\mathrm{R}$ at some time slot.

Once $P_{0}$ is received at $D$, via either direct or relay links, its age cycle starts and the level in $\Xi_{\mathrm{S}}$ increases by time until the next packet of $\mathrm{S}$ is successfully received at $\mathrm{D}$. During this time other packets of S may be accepted at $\mathrm{Q}$ and change the phase of $\Xi_{S}$ from $E$ to $F$ (recall that once $P_{0}$ is received at $\mathrm{D}, \mathrm{Q}$ becomes empty). Upon arrival of a new packet of $\mathrm{S}$ to $\mathrm{D}$, by using the auxiliary phase $\mathrm{T}, \Xi_{\mathrm{S}}$ restarts from level 1 to track the new packet.

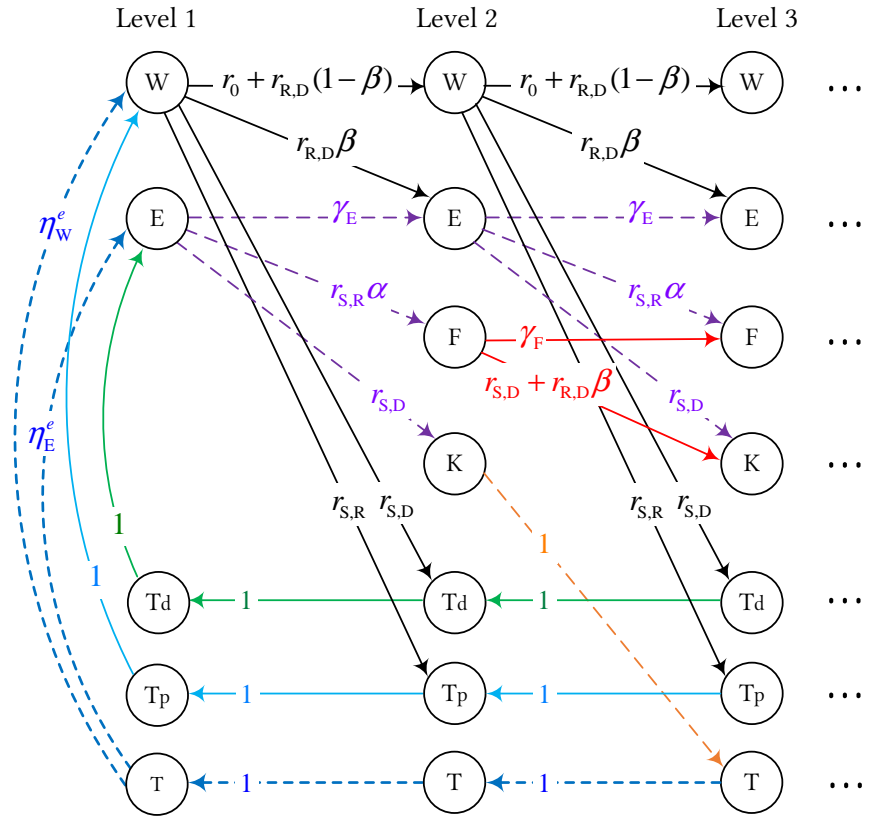

Fig. 3: The transition diagram of $\Xi_{\mathrm{S}}$, where $\gamma_{\mathrm{E}} \triangleq r_{0}+r_{\mathrm{R}, \mathrm{D}}+r_{\mathrm{S}, \mathrm{R}}(1-\alpha)$ and $\gamma_{\mathrm{F}} \triangleq r_{0}+r_{\mathrm{S}, \mathrm{R}}+r_{\mathrm{R}, \mathrm{D}}(1-\beta)$

Note that each packet of S may see Q empty or full when it departs $\mathrm{S}$. If $\mathrm{Q}$ is full and the new packet of $\mathrm{S}$ drops or replaces the existing packet in $Q$, the phases $T_{d}$ and $T_{p}$ restart $\Xi_{S}$ so that it models the journey of the new packet. Thus, when $\Xi_{\mathrm{S}}$ is restarted by means of phase $T$, it is going to track the journey of a packet which has entered the network when $\mathrm{Q}$ is empty. In that case, the packet enters $\mathrm{R}$ or $\mathrm{D}$, respectively by probabilities

$$
\eta_{\mathrm{W}}^{e} \triangleq \frac{r_{\mathrm{S}, \mathrm{R}} \alpha}{\left(r_{\mathrm{S}, \mathrm{D}}+r_{\mathrm{S}, \mathrm{R}} \alpha\right)}, \quad \eta_{\mathrm{E}}^{e} \triangleq \frac{r_{\mathrm{S}, \mathrm{D}}}{\left(r_{\mathrm{S}, \mathrm{D}}+r_{\mathrm{S}, \mathrm{R}} \alpha\right)},
$$

where $r_{\mathrm{S}, \mathrm{D}}+r_{\mathrm{S}, \mathrm{R}} \alpha$ is the probability of a successful transmission from $\mathrm{S}$ (to $\mathrm{R}$ or $\mathrm{D}$ ), provided that $\mathrm{Q}$ is empty. Also, by using the same reasoning as in Section IV-A, the phase $\mathrm{K}$ is introduced to $\Xi_{\mathrm{S}}$ to find $p_{\Lambda_{\mathrm{S}}}(n)$ as

$p_{\Lambda_{\mathrm{S}}}(n)=\lim _{\ell \rightarrow \infty} \operatorname{Pr}\left(L_{\ell}=n \mid P_{\ell}=\mathrm{K}\right)=\frac{\pi_{\mathrm{S}}(n, \mathrm{~K})}{\sum_{n=1}^{\infty} \pi_{\mathrm{S}}(n, \mathrm{~K})}$,

where $\pi_{\mathrm{S}}(x)$ is the steady state probability of being at state $x$ in $\Xi_{\mathrm{S}}$. Note that the auxiliary phases just aim to maintain the QBD structure of $\Xi_{\mathrm{S}}$ while restarting, also the goal of phase $\mathrm{K}$ is to find $p_{\Lambda_{\mathrm{S}}}(n)$ and the phase $\mathrm{W}$ tracks the packets whose age cycle has not yet begun. Thus, $p_{\Delta_{\mathrm{S}}}(n)$ is given by

$$
\begin{aligned}
p_{\Delta_{\mathrm{S}}}(n) & =\lim _{\ell \rightarrow \infty} \operatorname{Pr}\left(L_{\ell}=n \mid P_{\ell} \in\{\mathrm{E}, \mathrm{F}\}\right), \\
& =\frac{\pi_{\mathrm{S}}(n, \mathrm{E})+\pi_{\mathrm{S}}(n, \mathrm{~F})}{\sum_{n=1}^{\infty} \pi_{\mathrm{S}}(n, \mathrm{E})+\pi_{\mathrm{S}}(n, \mathrm{~F})} .
\end{aligned}
$$

\section{Joint PMf of AoI Sequences of the Processes}

To find $p(n, m)$, first note that $\operatorname{Pr}(\Psi(\ell)=(n, n))=0$ for $n \geqslant 1$. The reason is that $\Psi(\ell)=(n, n)$ conveys that the last received packets of $S$ and $R$ have been accepted at $D$ simultaneously $n$ time slots ago, which is not probable due to the adopted collision model. Accordingly, we just need to investigate the cases $\Delta_{\mathrm{S}}(\ell)<\Delta_{\mathrm{R}}(\ell)$ and $\Delta_{\mathrm{S}}(\ell)>\Delta_{\mathrm{R}}(\ell)$. 
TABLE II: Description of phases in QBD models

\begin{tabular}{|c|c|}
\hline Phase & Description \\
\hline $\mathrm{E}$ & $\mathrm{P}_{0}{ }^{(\mathrm{a})}$ is received successfully at $\mathrm{D}$ while $\mathrm{Q}$ is empty. \\
\hline $\mathrm{F}$ & $\begin{array}{l}\mathrm{P}_{0}{ }^{(a)} \text { is received successfully at } \mathrm{D} \text { while another packet is } \\
\text { waiting at } \mathrm{Q} \text {. }\end{array}$ \\
\hline $\mathrm{W}$ & $\mathrm{P}_{0}$ is waiting at $\mathrm{Q}$. \\
\hline $\begin{array}{c}\text { F-io } \\
\text { (in } \Xi_{\mathrm{S}>\mathrm{R}}^{f} \text { ) }\end{array}$ & $\begin{array}{l}\mathrm{P}_{0} \text { has been received at } \mathrm{D} \text { while } \mathrm{Q} \text { was full, then another } \\
\text { packet of } \mathrm{S} \text { has replaced the existing packet in } \mathrm{Q} \text {. }\end{array}$ \\
\hline $\begin{array}{c}\text { F-oo } \\
\text { (in } \Xi_{\mathrm{S}>\mathrm{R}}^{f} \text { ) }\end{array}$ & $\begin{array}{l}\mathrm{P}_{0} \text { has been received at } \mathrm{D} \text { while } \mathrm{Q} \text { was full, then the } \\
\text { waiting packet in } \mathrm{Q} \text { has been delivered to } \mathrm{D} \text {, then a new } \\
\text { packet of } \mathrm{S} \text { is accepted at } \mathrm{Q} \text {. }\end{array}$ \\
\hline $\mathrm{T}$ & Transition phase, used if $\mathrm{P}_{0}$ is not replaced or dropped. \\
\hline $\mathrm{T}_{\mathrm{p}}\left(\mathrm{T}_{\mathrm{d}}\right)$ & Transition phase (in $\Xi_{S}$ ), used if $P_{0}$ is replaced (dropped). \\
\hline $\mathrm{T}_{\mathrm{e}}\left(\mathrm{T}_{\mathrm{f}}\right)$ & $\begin{array}{l}\text { Transition phase (in } \Xi_{R} \text { ), used if the new packet is received } \\
\text { when Q is empty (full). }\end{array}$ \\
\hline $\mathrm{K}$ & The phase to account for $\Lambda_{\mathrm{S}}(\ell)$ in $\Xi_{\mathrm{S}}$. \\
\hline$K_{e}\left(K_{f}\right)$ & $\begin{array}{l}\text { The phase to account for } \Lambda_{R}(\ell) \text { in } \Xi_{R} \text {, where } Q \text { is empty (full) } \\
\text { upon the arrival of the new packet. }\end{array}$ \\
\hline
\end{tabular}

(a) $\mathrm{P}_{0}$ is the most recent received packet of $\mathrm{S}(\mathrm{R})$ in the scenarios where $\Delta_{\mathrm{S}}(\ell)<\Delta_{\mathrm{R}}(\ell)\left(\Delta_{\mathrm{S}}(\ell)>\Delta_{\mathrm{R}}(\ell)\right)$.

To that end, at first in Section V-A we restrict our attention only to the set of time slots where $\Delta_{S}(\ell)<\Delta_{R}(\ell)$ and divide that into two subsets, namely $T_{\mathrm{S}<\mathrm{R}}^{e}$ and $T_{\mathrm{S}<\mathrm{R}}^{f}$, based on the state of $\mathrm{Q}$ in time slot $\ell-n$. We find $\lim \operatorname{Pr}(\Psi(\ell)=$ $(n, m))$ when $\ell$ is in each of these subsets. Following that, in Section V-B, we draw attention just to the time slots where $\Delta_{\mathrm{S}}(\ell)>\Delta_{\mathrm{R}}(\ell)$ and classify them into three subsets, named as $T_{\mathrm{S}>\mathrm{R}}^{e}, T_{\mathrm{S}>\mathrm{R}}^{f-o}$ and $T_{\mathrm{S}>\mathrm{R}}^{f-i o}$, based on the identity of the packet in $\mathrm{Q}$ in time slot $\ell-m$. Then $\lim _{\ell \rightarrow \infty} \operatorname{Pr}(\Psi(\ell)=(n, m))$ is found conditioned on $\ell$ being in each of these three subsets. The key features that help us to find $p(n, m)$, are the independency properties of $\Psi(\ell)$ once $\ell \in T$ where $T$ is each of the above mentioned five subsets of time slots, which are proven in Lemmas 1 and 2. Hereafter, when $\ell \in T$ we state that $\Psi(\ell)$ is in stage $T$. In Secs. V-A and V-B, we derive $\lim _{\ell \rightarrow \infty} \operatorname{Pr}(\Psi(\ell)=(n, m) \mid \ell \in T)$, while the stationary distribution of stages is found in Sec. V-C to obtain $p(n, m)$.

\section{A. Case $\Delta_{\mathrm{S}}(\ell)<\Delta_{\mathrm{R}}(\ell)$}

In this section $p(n, m)$ is found for $m>n \geqslant 1$, where $m=n+k$ for $n, k \geqslant 1$. Consider some time slot $\ell$, at the beginning of which $\Delta_{\mathrm{S}}(\ell)=n$ and $\Delta_{\mathrm{R}}(\ell)=n+k$ (see Fig. 4). In this case, the last received packet of $\mathrm{R}$ has been transmitted at time slot $\ell-n-k$ while the last received packet of $\mathrm{S}$ has been generated $k$ time slots after that, at $\ell-n$. This packet of $\mathrm{S}$, namely $\mathrm{P}_{0}$, may have traveled through the direct link to $\mathrm{D}$ or it may have been accepted at $\mathrm{Q}$ at $\ell-n$ and then delivered to $\mathrm{D}$ at some time slot after that (before $\ell$ ). For $\Delta_{\mathrm{S}}(\ell)=n$, $\mathrm{P}_{0}$ should not be replaced or dropped during the time interval $[\ell-n+1, \ell]$. Also, no other packet of $\mathrm{S}$ should be received at $\mathrm{D}$ after receiving $\mathrm{P}_{0}$. Let's call that event, maintaining $\mathrm{P}_{0}$ in the interval $[\ell-n+1, \ell]$. Using similar expression, the last received packet of $\mathrm{R}\left(\mathrm{P}^{\mathrm{R}}\right.$ in Fig. 4) should be maintained in the interval $[\ell-n-k+1, \ell]$ to assure $\Delta_{\mathrm{R}}(\ell)=n+k$.

Accordingly, having $q(\ell)$ to show the state of $\mathrm{Q}$ at time slot $\ell$ (i.e., $q(\ell)=0$ or 1 , if $\mathrm{Q}$ is empty or full at the onset of $\ell$,

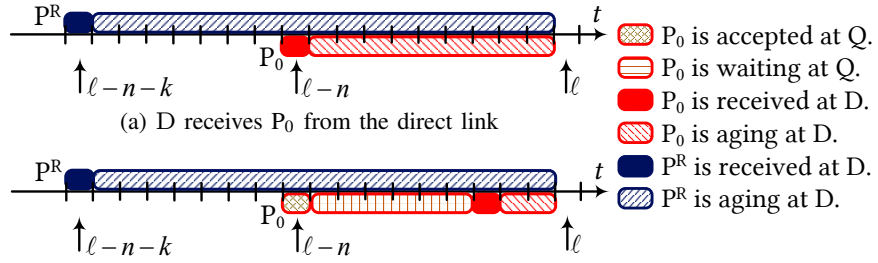

(b) $\mathrm{D}$ receives $\mathrm{P}_{0}$ from the relay link

Fig. 4: Possible scenarios to have $\Delta_{S}(\ell)<\Delta_{R}(\ell)$

resp.), we can write $\operatorname{Pr}(\Psi(\ell)=(n, n+k))$ as

$$
\begin{aligned}
& \operatorname{Pr}(\Psi(\ell)=(n, n+k))= \\
& \operatorname{Pr}\left(\Psi(\ell)=(n, n+k) \mid \Delta_{\mathrm{R}}(\ell-n)=k, q(\ell-n)=0\right) \\
& \quad \times \operatorname{Pr}\left(\Delta_{\mathrm{R}}(\ell-n)=k, q(\ell-n)=0\right)+ \\
& \operatorname{Pr}\left(\Psi(\ell)=(n, n+k) \mid \Delta_{\mathrm{R}}(\ell-n)=k, q(\ell-n)=1\right) \\
& \quad \times \operatorname{Pr}\left(\Delta_{\mathrm{R}}(\ell-n)=k, q(\ell-n)=1\right) .
\end{aligned}
$$

Considering (9), letting $\ell \rightarrow \infty$ in (16) yields $p(n, n+k)$. While Lemma 1 narrows the way to find the steady state conditional probabilities in (16), for $\lim _{\ell \rightarrow \infty} \operatorname{Pr}\left(\Delta_{\mathrm{R}}(\ell-n)=k, q(\ell-n\right.$ $=0)$ and $\lim _{\ell \rightarrow \infty} \operatorname{Pr}\left(\Delta_{\mathrm{R}}(\ell-n) \stackrel{\ell \rightarrow \infty}{=} k, q(\ell-n)=1\right)$ we have

$$
\begin{aligned}
P_{\Delta_{\mathrm{R}}}^{\dashv e}(k) & \triangleq \lim _{\ell \rightarrow \infty} \operatorname{Pr}\left(\Delta_{\mathrm{R}}(\ell-n)=k, q(\ell-n)=0\right) \\
& =\frac{\pi_{\mathrm{R}}(k, \mathrm{E})}{\sum_{k=1}^{\infty} \pi_{\mathrm{R}}(k, \mathrm{E})+\pi_{\mathrm{R}}(k, \mathrm{~F})}, \\
P_{\Delta_{\mathrm{R}}}^{\dashv f}(k) & \triangleq \lim _{\ell \rightarrow \infty} \operatorname{Pr}\left(\Delta_{\mathrm{R}}(\ell-n)=k, q(\ell-n)=1\right) \\
& =\frac{\pi_{\mathrm{R}}(k, \mathrm{~F})}{\sum_{k=1}^{\infty} \pi_{\mathrm{R}}(k, \mathrm{E})+\pi_{\mathrm{R}}(k, \mathrm{~F})},
\end{aligned}
$$

where $\pi_{\mathrm{R}}$ is the stationary distribution of $\Xi_{\mathrm{R}}$, detailed in Section IV-A. In fact, $P_{\Delta_{\mathrm{R}}}^{\dashv e}(k)$ (and also $P_{\Delta_{\mathrm{R}}}^{\dashv f}(k)$ ) is the steady state probability of the event that $\mathrm{Q}$ is empty (full, resp.), once the last received packet of $\mathrm{R}$ is aged for $k$ time slots at $\mathrm{D}$.

Lemma 1: The conditional probabilities $\operatorname{Pr}(\Psi(\ell)=(n, n+$ $\left.k) \mid \Delta_{\mathrm{R}}(\ell-n)=k, q(\ell-n)=0\right)$ and $\operatorname{Pr}(\Psi(\ell)=(n, n+$ k) $\left.\mid \Delta_{\mathrm{R}}(\ell-n)=k, q(\ell-n)=1\right)$ are independent of $k$.

Proof: Consider the scenarios depicted in Fig. 4. Note that maintaining the packet of $\mathrm{R}$ in the interval $[\ell-n-k+$ $1, \ell-n-1]$ affects the probability of maintaining packets of $\mathrm{S}$ and $\mathrm{R}$ in the interval $[\ell-n+1, \ell]$ just by changing the state of $\mathrm{Q}$ at the onset of time slot $\ell-n$. That is, if we know that $q(\ell-n)=0$, the probability of maintaining packets of $\mathrm{S}$ and $\mathrm{R}$ in the time interval $[\ell-n+1, \ell]$ does not depend on how long the packet of $\mathrm{R}$ has been maintained before $\ell-n$. Therefore, the independency of $\operatorname{Pr}\left(\Psi(\ell)=(n, n+k) \mid \Delta_{\mathrm{R}}(\ell-n)=\right.$ $k, q(\ell-n)=0)$ of $k$ is deduced. Applying similar reasoning results in the independency of $\operatorname{Pr}\left(\Psi(\ell)=(n, n+k) \mid \Delta_{\mathrm{R}}(\ell-\right.$ $n)=k, q(\ell-n)=1)$ of $k$ and concludes the lemma.

According to Lemma 1 and by using (16) and (17), we have

$$
p(n, n+k)=C_{\mathrm{S}<\mathrm{R}}^{e}(n) P_{\Delta_{\mathrm{R}}}^{\dashv e}(k)+C_{\mathrm{S}<\mathrm{R}}^{f}(n) P_{\Delta_{\mathrm{R}}}^{\dashv f}(k),
$$




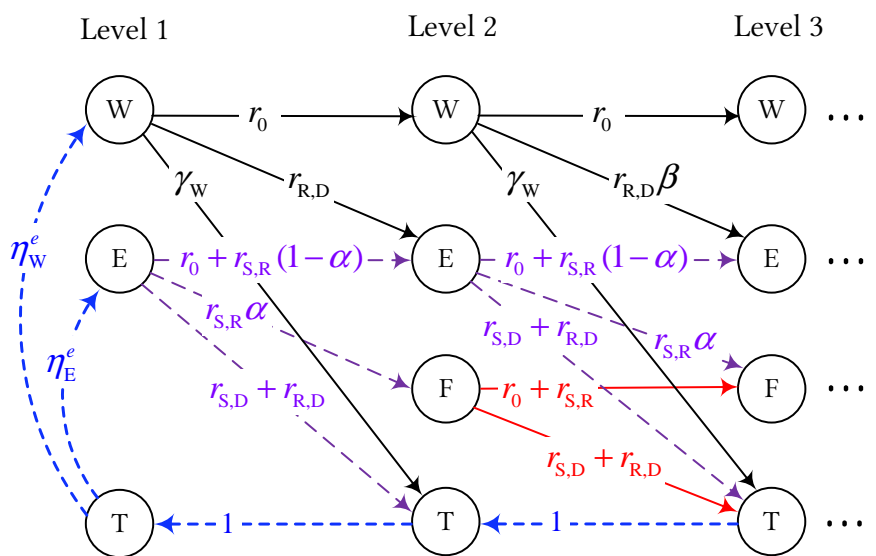

Fig. 5: The transition diagram of $\Xi_{\mathrm{S}<\mathrm{R}}^{e}, \gamma_{\mathrm{W}} \triangleq r_{\mathrm{S}, \mathrm{D}}+r_{\mathrm{S}, \mathrm{R}}+r_{\mathrm{R}, \mathrm{D}}(1-\beta)$

where

$$
\begin{aligned}
C_{\mathrm{S}<\mathrm{R}}^{e}(n) \triangleq \lim _{\ell \rightarrow \infty} \operatorname{Pr}(\Psi(\ell)= & (n, n+k) \mid \Delta_{\mathrm{R}}(\ell-n)=k, \\
& q(\ell-n)=0), \\
C_{\mathrm{S}<\mathrm{R}}^{f}(n) \triangleq \lim _{\ell \rightarrow \infty} \operatorname{Pr}(\Psi(\ell)= & (n, n+k) \mid \Delta_{\mathrm{R}}(\ell-n)=k, \\
& q(\ell-n)=1) .
\end{aligned}
$$

To find $C_{\mathrm{S}<\mathrm{R}}^{e}(n)$ and $C_{\mathrm{S}<\mathrm{R}}^{f}(n)$, we propose the QBD type Markov chains $\Xi_{\mathrm{S}<\mathrm{R}}^{e}$ and $\Xi_{\mathrm{S}<\mathrm{R}}^{f}$, respectively, which are very similar to $\Xi_{\mathrm{S}}$. To that end, let $T_{\mathrm{S}<\mathrm{R}}^{e}\left(\right.$ and $T_{\mathrm{S}<\mathrm{R}}^{f}$ ) denote the set of all time slots where $\Delta_{\mathrm{S}}(\ell)<\Delta_{\mathrm{R}}(\ell)$ with the condition that $\mathrm{Q}$ has been empty (full, resp.) upon entrance of the last received packet of $\mathrm{S}$, i.e., $\mathrm{P}_{0}$. In fact, $T_{\mathrm{S}<\mathrm{R}}^{e}$ and $T_{\mathrm{S}<\mathrm{R}}^{f}$ indicate the first two stages that are described earlier. Being confined to $\ell \in T_{\mathrm{S}<\mathrm{R}}^{e}$ (and $\ell \in T_{\mathrm{S}<\mathrm{R}}^{f}$ ), $\Xi_{\mathrm{S}<\mathrm{R}}^{e}\left(\Xi_{\mathrm{S}<\mathrm{R}}^{f}\right.$, resp.) models the age cycle of $\mathrm{P}_{0}$ until another packet of $\mathrm{S}$ or $\mathrm{R}$ is received at D. Table II describes the phases of $\Xi_{\mathrm{S}<\mathrm{R}}^{e}$ and $\Xi_{\mathrm{S}<\mathrm{R}}^{f}$.

Fig. 5 depicts $\Xi_{\mathrm{S}<\mathrm{R}}^{e}$, where $\mathrm{P}_{0}$ starts its journey from level 1 at phase $\mathrm{E}$ or $\mathrm{W}$, if it is delivered to $\mathrm{D}$ or accepted at $\mathrm{Q}$, respectively. Level of $\Xi_{\mathrm{S}<\mathrm{R}}^{e}$ is incremented by 1 at each time slot, if $\mathrm{P}_{0}$ is not dropped or replaced and also another packet of $\mathrm{S}$ or $\mathrm{R}$ is not received at $\mathrm{D}$. Meanwhile, once $\mathrm{P}_{0}$ waits at $\mathrm{Q}$, there may be a phase transition from $\mathrm{W}$ to $\mathrm{E}$, due to delivery of $\mathrm{P}_{0}$ to $\mathrm{D}$. Moreover, once $\mathrm{P}_{0}$ is received at $\mathrm{D}$, another packet of $\mathrm{S}$ may be accepted at $\mathrm{Q}$, which changes the phase from $\mathrm{E}$ to $F$. Anyway, once $P_{0}$ is dropped or replaced or a new packet of $\mathrm{S}$ or $\mathrm{R}$ arrives to $\mathrm{D}, \Xi_{\mathrm{S}<\mathrm{R}}^{e}$ is restarted (through the phase $\mathrm{T}$ ) to model the age cycle of another packet of $\mathrm{S}$ that sees empty $\mathrm{Q}$ while entering the network. The new packet is accepted at $\mathrm{Q}$ with probability $\eta_{\mathrm{W}}^{e}$ or received at D directly with probability $\eta_{\mathrm{E}}^{e}$, where $\eta_{\mathrm{W}}^{e}$ and $\eta_{\mathrm{E}}^{e}$ are given by (13).

Similar to $\Xi_{\mathrm{S}}$, since the auxiliary phase $\mathrm{T}$ is introduced just to maintain the QBD form of $\Xi_{\mathrm{S}<\mathrm{R}}^{e}$, and also phase $\mathrm{W}$ only tracks $\mathrm{P}_{0}$ until it is received at $\mathrm{D}, \mathrm{P}_{0}$ is actually aged only at phases $\mathrm{E}$ and $\mathrm{F}$. Thus, if $\pi_{\mathrm{S}<\mathrm{R}}^{e}(x)$ denotes the stationary distribution of $\Xi_{\mathrm{S}<\mathrm{R}}^{e}$ at state $x$, we have

$$
\begin{aligned}
& \lim _{\ell \rightarrow \infty} \operatorname{Pr}\left(\Delta_{\mathrm{S}}(\ell)=n \mid \ell \in T_{\mathrm{S}<\mathrm{R}}^{e}\right)= \\
& \lim _{\ell \rightarrow \infty} \operatorname{Pr}\left(L_{\ell}=n \mid P_{\ell} \in\{\mathrm{E}, \mathrm{F}\}\right)= \\
& \quad \frac{\pi_{\mathrm{S}<\mathrm{R}}^{e}(n, \mathrm{E})+\pi_{\mathrm{S}<\mathrm{R}}^{e}(n, \mathrm{~F})}{\sum_{n=1}^{\infty} \pi_{\mathrm{S}<\mathrm{R}}^{e}(n, \mathrm{E})+\pi_{\mathrm{S}<\mathrm{R}}^{e}(n, \mathrm{~F})}, \quad n \geqslant 1,
\end{aligned}
$$

$\begin{array}{lll}\text { Level } 1 & \text { Level } 2 & \text { Level } 3\end{array}$

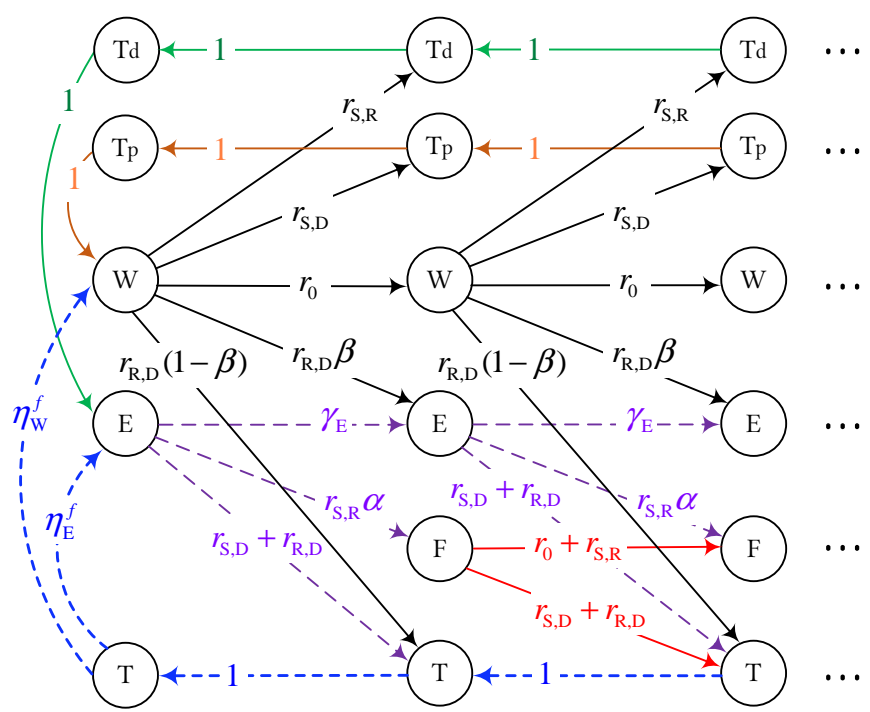

Fig. 6: The transition diagram of $\Xi_{\mathrm{S}<\mathrm{R}}^{f}$, where $\gamma_{\mathrm{E}} \triangleq r_{0}+r_{\mathrm{S}, \mathrm{R}}(1-\alpha)$

where we assume $\pi_{\mathrm{S}<\mathrm{R}}^{e}(1, \mathrm{~F})=0$. On the other hand,

$$
\begin{aligned}
& \operatorname{Pr}\left(\Delta_{\mathrm{S}}(\ell)=n \mid \ell \in T_{\mathrm{S}<\mathrm{R}}^{e}\right)=\frac{\operatorname{Pr}\left(\Delta_{\mathrm{S}}(\ell)=n, \ell \in T_{\mathrm{S}<\mathrm{R}}^{e}\right)}{\operatorname{Pr}\left(\ell \in T_{\mathrm{S}<\mathrm{R}}^{e}\right)} \\
& =\frac{\sum_{k=1}^{\infty} \operatorname{Pr}(\Psi(\ell)=(n, n+k), q(\ell-n)=0)}{\operatorname{Pr}\left(\ell \in T_{\mathrm{S}<\mathrm{R}}^{e}\right)}
\end{aligned}
$$

Now, by letting $\ell \rightarrow \infty$ and using (17a) and (19a), we find

$$
\begin{aligned}
\lim _{\ell \rightarrow \infty} \operatorname{Pr}\left(\Delta_{\mathrm{S}}(\ell)=n \mid \ell \in T_{\mathrm{S}<\mathrm{R}}^{e}\right) \\
\quad=C_{\mathrm{S}<\mathrm{R}}^{e}(n) \frac{\sum_{k=1}^{\infty} p_{\Delta_{\mathrm{R}}}^{\dashv e}(k)}{\tau_{\mathrm{S}<\mathrm{R}}^{e}}=C_{\mathrm{S}<\mathrm{R}}^{e}(n) \frac{q^{e}}{\tau_{\mathrm{S}<\mathrm{R}}^{e}},
\end{aligned}
$$

where $\tau_{\mathrm{S}<\mathrm{R}}^{e} \triangleq \lim _{\ell \rightarrow \infty} \operatorname{Pr}\left(\ell \in T_{\mathrm{S}<\mathrm{R}}^{e}\right)$ is the steady state probability of $\Psi(\ell)$ to be in stage $T_{\mathrm{S}<\mathrm{R}}^{e}$. Also, $q^{e}$ being the steady state probability of $\mathrm{Q}$ to be empty, is found from the Markov chain in Fig. 7, where the states indicate $q(\ell)$; that is,

$$
q^{e}=1-q^{f}=\frac{r_{\mathrm{S}, \mathrm{D}}+r_{\mathrm{R}, \mathrm{D}} \beta}{r_{\mathrm{S}, \mathrm{D}}+r_{\mathrm{R}, \mathrm{D}} \beta+r_{\mathrm{S}, \mathrm{R}} \alpha},
$$

where $q^{f}$ indicates the probability of finding a full $\mathrm{Q}$ in the steady state. Therefore, once $\tau_{\mathrm{S}<\mathrm{R}}^{e}$ is known (given in Section V-C) $C_{\mathrm{S}<\mathrm{R}}^{e}(n)$ is determined by using (20) and (22).

A similar approach is followed to find $C_{\mathrm{S}<\mathrm{R}}^{f}(n)$. Illustrated in Fig. $6, \Xi_{\mathrm{S}<\mathrm{R}}^{f}$ models the age cycle of $\mathrm{P}_{0}$ provided that $\ell \in T_{\mathrm{S}<\mathrm{R}}^{f}$. More specifically, once $\mathrm{P}_{0}$ replaces the existing packet in $\mathrm{Q}$ or it is received at $\mathrm{D}$ directly (and drops the waiting packet in Q), its journey in $\Xi_{\mathrm{S}<\mathrm{R}}^{f}$ starts from level 1 at phase $\mathrm{W}$ or $\mathrm{E}$, respectively, and evolves level by level as in $\Xi_{\mathrm{S}<\mathrm{R}}^{e}$. However, if $\mathrm{P}_{0}$ is replaced (or dropped) by another packet of $\mathrm{S}$, the auxiliary phase $\mathrm{T}_{\mathrm{p}}\left(\mathrm{T}_{\mathrm{d}}\right.$, resp.) restarts $\Xi_{\mathrm{S}<\mathrm{R}}^{f}$ from level 1 at phase $\mathrm{W}$ (E, resp.). Moreover, since $\ell \in T_{\mathrm{S}<\mathrm{R}}^{f}$, a new packet of $\mathrm{S}$ enters $\mathrm{Q}$ with probability $\eta_{\mathrm{W}}^{f}$ or is received directly at $\mathrm{D}$ with probability $\eta_{\mathrm{E}}^{f}$, where

$$
\eta_{\mathrm{W}}^{f} \triangleq \frac{r_{\mathrm{S}, \mathrm{R}}}{\left(r_{\mathrm{S}, \mathrm{D}}+r_{\mathrm{S}, \mathrm{R}}\right)}, \quad \eta_{\mathrm{E}}^{f} \triangleq \frac{r_{\mathrm{S}, \mathrm{D}}}{\left(r_{\mathrm{S}, \mathrm{D}}+r_{\mathrm{S}, \mathrm{R}}\right)},
$$




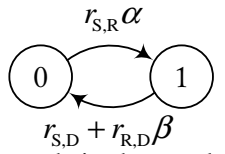

Fig. 7: The Markov chain that models the state of $Q$

in which $r_{\mathrm{S}, \mathrm{D}}+r_{\mathrm{S}, \mathrm{R}}$ is the probability of successful departure from $\mathrm{S}$, when $\mathrm{Q}$ is full. Now by solving $\Xi_{\mathrm{S}<\mathrm{R}}^{f}$ we find

$$
\begin{aligned}
& \lim _{\ell \rightarrow \infty} \operatorname{Pr}\left(\Delta_{\mathrm{S}}(\ell)=n \mid \ell \in T_{\mathrm{S}<\mathrm{R}}^{f}\right)= \\
& \lim _{\ell \rightarrow \infty} \operatorname{Pr}\left(L_{\ell}=n \mid P_{\ell} \in\{\mathrm{E}, \mathrm{F}\}\right)= \\
& \quad \frac{\pi_{\mathrm{S}<\mathrm{R}}^{f}(n, \mathrm{E})+\pi_{\mathrm{S}<\mathrm{R}}^{f}(n, \mathrm{~F})}{\sum_{n=1}^{\infty} \pi_{\mathrm{S}<\mathrm{R}}^{f}(n, \mathrm{E})+\pi_{\mathrm{S}<\mathrm{R}}^{f}(n, \mathrm{~F})}, \quad n \geqslant 1,
\end{aligned}
$$

in which $\pi_{\mathrm{S}<\mathrm{R}}^{f}(x)$ is the stationary distribution of $\Xi_{\mathrm{S}<\mathrm{R}}^{f}$ at state $x$, and $\pi_{\mathrm{S}<\mathrm{R}}^{f}(1, \mathrm{~F})=0$. Doing similar manipulation to (21) and (22), results in

$$
\lim _{\ell \rightarrow \infty} \operatorname{Pr}\left(\Delta_{\mathrm{S}}(\ell)=n \mid \ell \in T_{\mathrm{S}<\mathrm{R}}^{f}\right)=C_{\mathrm{S}<\mathrm{R}}^{f}(n) \frac{q^{f}}{\tau_{\mathrm{S}<\mathrm{R}}^{f}},
$$

in which $q^{f}$ is given by (23) and $\tau_{\mathrm{S}<\mathrm{R}}^{f} \triangleq \lim _{\ell \rightarrow \infty} \operatorname{Pr}\left(\ell \in T_{\mathrm{S}<\mathrm{R}}^{f}\right)$.

\section{B. Case $\Delta_{\mathrm{S}}(\ell)>\Delta_{\mathrm{R}}(\ell)$}

To find $p(n, m)$ for $n>m \geqslant 1$, let $n=m+k$ for $m, k \geqslant 1$. To have $\Psi(\ell)=(m+k, m)$ at time slot $\ell$, a packet of S , e.g., $\mathrm{P}^{\mathrm{S}}$, leaves this node at time slot $\ell-m-k$ and is maintained till the time $\ell$. Meanwhile the last received packet of $\mathrm{R}$, e.g., $\mathrm{P}_{0}$, is generated at time slot $\ell-m$. Fig. 8 illustrates three possible scenarios that may result in $\Psi(\ell)=(m+k, m)$. In the first scenario $\mathrm{P}_{0}$ enters $\mathrm{D}$ when $\mathrm{Q}$ is empty, while in the $2^{\text {nd }}$ and $3^{\text {rd }}$ scenarios $Q$ is full upon the arrival of $\mathrm{P}_{0}$. In the $2^{\text {nd }}$ scenario, $\mathrm{P}^{\mathrm{S}}$ is delivered to $\mathrm{D}$ before time slot $\ell-m$ and after that another packet of $\mathrm{S}$ is accepted by $\mathrm{R}$, which is waiting in $\mathrm{Q}$ when $\mathrm{P}_{0}$ is received at $\mathrm{D}$. However, in the $3^{\text {rd }}$ scenario, the waiting packet in $\mathrm{Q}$ at time slot $\ell-m$ is $\mathrm{P}^{\mathrm{S}}$. This makes the $3^{\text {rd }}$ scenario different from all other scenarios in Figs. 4 and 8 , in the way that in all of the other scenarios the packet which arrives later to $\mathrm{D}$ has smaller age at $\mathrm{D}$, however in the $3^{\text {rd }}$ scenario, once $\mathrm{P}^{\mathrm{S}}$ is received at $\mathrm{D}$, its age is larger than the age of $\mathrm{P}_{0}$, even though $\mathrm{P}^{\mathrm{S}}$ has arrived later than $\mathrm{P}_{0}$. Hence, we refer to the $3^{\text {rd }}$ scenario in Fig. 8 as the out of order delivery scenario (indicated by $-o o$ in the superscripts of variables) so as to distinguish it from the $2^{\text {nd }}$ scenario which is called the in order delivery scenario (indicated by -io in the superscripts).

Henceforth, $\operatorname{Pr}(\Psi(\ell)=(m+k, m))$ is written as

$$
\begin{aligned}
& \operatorname{Pr}(\Psi(\ell)=(m+k, m))= \\
& \operatorname{Pr}\left(\Psi(\ell)=(m+k, m) \mid \Delta_{\mathrm{S}}(\ell-m)=k, q(\ell-m)=0\right) \\
& \quad \times \operatorname{Pr}\left(\Delta_{\mathrm{S}}(\ell-m)=k, q(\ell-m)=0\right)+ \\
& \operatorname{Pr}\left(\Psi(\ell)=(m+k, m) \mid \Delta_{\mathrm{S}}(\ell-m)=k, q(\ell-m)=1\right) \\
& \quad \times \operatorname{Pr}\left(\Delta_{\mathrm{S}}(\ell-m)=k, q(\ell-m)=1\right)+ \\
& \operatorname{Pr}\left(\Psi(\ell)=(m+k, m) \mid \Omega_{\mathrm{S}}(\ell-m)=k, q(\ell-m)=1\right) \\
& \quad \times \operatorname{Pr}\left(\Omega_{\mathrm{S}}(\ell-m)=k, q(\ell-m)=1\right)
\end{aligned}
$$

where $\Omega_{\mathrm{S}}(\ell)$ indicates the AoI sequence of $\mathrm{S}$ monitored at $\mathrm{R}$, i.e., the elapsed time since the generation instance of the last

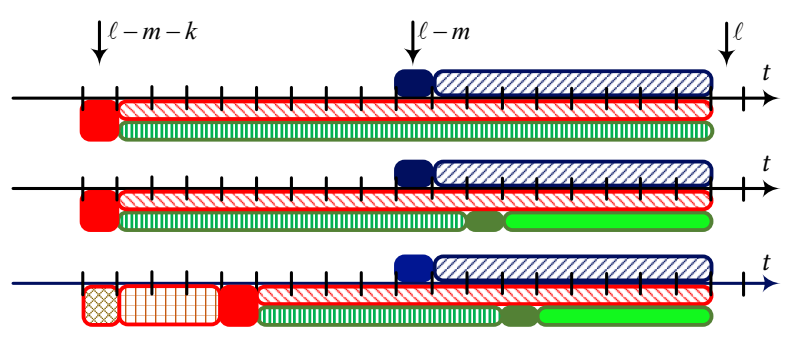

(a) Scenario 1: $P_{0}$ arrives when $Q$ is empty.

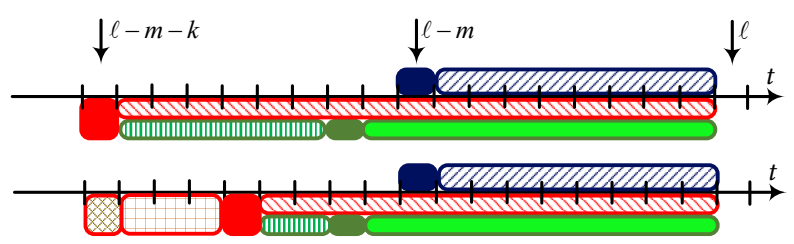

(b) Scenario 2: $\mathrm{P}_{0}$ arrives when $\mathrm{Q}$ is full with a packet other than $\mathrm{P}^{\mathrm{S}}$.

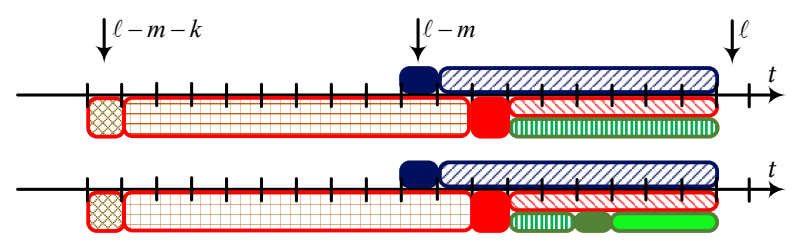

(c) Scenario 3: $\mathrm{P}_{0}$ arrives when $\mathrm{P}^{\mathrm{S}}$ is waiting in $\mathrm{Q}$.

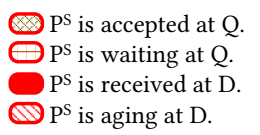

$\mathrm{P}_{0}$ is received at $\mathrm{D}$.

$0 \mathrm{P}_{0}$ is aging at $\mathrm{D}$.

(IIII) $Q$ is empty.

Another packet of $S$ is accepted at $Q$.

Another packet of S is waiting at Q.

Fig. 8: Possible scenarios to have $\Delta_{S}(\ell)>\Delta_{R}(\ell)$

received packet of $\mathrm{S}$ at $\mathrm{R}$. The following lemma shows that the conditional probabilities in (27) are independent of $k$.

Lemma 2: The conditional probabilities $\operatorname{Pr}(\Psi(\ell)=(m+$ $\left.k, m) \mid \Delta_{\mathrm{S}}(\ell-m)=k, q(\ell-m)=0\right), \operatorname{Pr}(\Psi(\ell)=(m+$ $\left.k, m) \mid \Delta_{\mathrm{S}}(\ell-m)=k, q(\ell-m)=1\right)$ and $\operatorname{Pr}(\Psi(\ell)=(m+$ $\left.k, m) \mid \Omega_{\mathrm{S}}(\ell-m)=k, q(\ell-m)=1\right)$ are independent of $k$.

Proof: The proof follows the same reasoning as in the proof of Lemma 1 and is left for the respectful reader.

Let $\ell \rightarrow \infty$ in (27). Using Lemma 2 gives

$$
\begin{aligned}
p(m+k, m) & =C_{\mathrm{S}>\mathrm{R}}^{e}(m) P_{\Delta_{\mathrm{S}}}^{\dashv e}(k)+C_{\mathrm{S}>\mathrm{R}}^{f-i o}(m) P_{\Delta_{\mathrm{S}}}^{\dashv f}(k) \\
& +C_{\mathrm{S}>\mathrm{R}}^{f-o o}(m) P_{\Omega_{\mathrm{S}}}^{\dashv f}(k)
\end{aligned}
$$

in which

$$
\begin{aligned}
C_{\mathrm{S}>\mathrm{R}}^{e}(m) \triangleq \lim _{\ell \rightarrow \infty} \operatorname{Pr}(\Psi(\ell)= & (m+k, m) \mid \Delta_{\mathrm{S}}(\ell-m)=k \\
& q(\ell-m)=0) \\
C_{\mathrm{S}>\mathrm{R}}^{f-i o}(m) \triangleq \lim _{\ell \rightarrow \infty} \operatorname{Pr}(\Psi(\ell)= & (m+k, m) \mid \Delta_{\mathrm{S}}(\ell-m)=k \\
& q(\ell-m)=1) \\
C_{\mathrm{S}>\mathrm{R}}^{f-o o}(m) \triangleq \lim _{\ell \rightarrow \infty} \operatorname{Pr}(\Psi(\ell)= & (m+k, m) \mid \Omega_{\mathrm{S}}(\ell-m)= \\
& q(\ell-m)=1)
\end{aligned}
$$

and also

$$
\begin{aligned}
P_{\Delta_{\mathrm{S}}}^{\dashv e}(k) & \triangleq \lim _{\ell \rightarrow \infty} \operatorname{Pr}\left(\Delta_{\mathrm{S}}(\ell-m)=k, q(\ell-m)=0\right) \\
& =\frac{\pi_{\mathrm{S}}(k, \mathrm{E})}{\sum_{k=1}^{\infty} \pi_{\mathrm{S}}(k, \mathrm{E})+\pi_{\mathrm{S}}(k, \mathrm{~F})}
\end{aligned}
$$




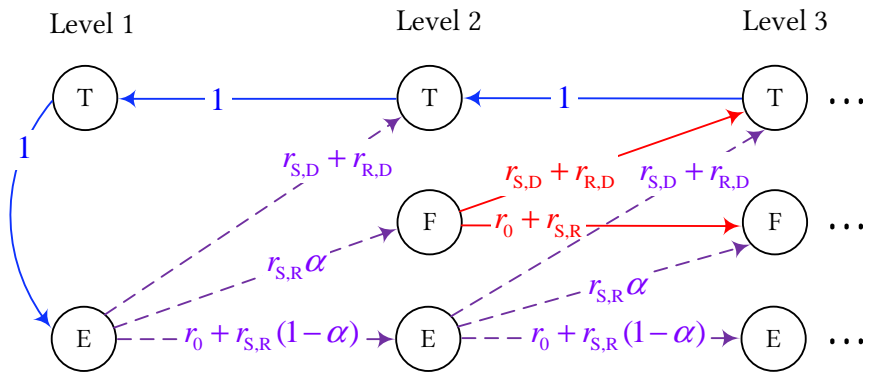

Fig. 9: The transition diagram of $\Xi_{\mathrm{S}>\mathrm{R}}^{e}$

$$
\begin{aligned}
P_{\Delta_{\mathrm{S}}}^{\dashv f}(k) & \triangleq \lim _{\ell \rightarrow \infty} \operatorname{Pr}\left(\Delta_{\mathrm{S}}(\ell-m)=k, q(\ell-m)=1\right) \\
& =\frac{\pi_{\mathrm{S}}(k, \mathrm{~F})}{\sum_{k=1}^{\infty} \pi_{\mathrm{S}}(k, \mathrm{E})+\pi_{\mathrm{S}}(k, \mathrm{~F})}, \\
P_{\Omega_{\mathrm{S}}}^{\dashv f}(k) & \triangleq \lim _{\ell \rightarrow \infty} \operatorname{Pr}\left(\Omega_{\mathrm{S}}(\ell-m)=k, q(\ell-m)=1\right) \\
& =\frac{\pi_{\mathrm{S}}(k, \mathrm{~W})}{\sum_{k=1}^{\infty} \pi_{\mathrm{S}}(k, \mathrm{~W})},
\end{aligned}
$$

where $\pi_{\mathrm{S}}(x)$ is the stationary distribution of $\Xi_{\mathrm{S}}$ explained in Sec. IV-B. Note that $P_{\Delta_{\mathrm{S}}}^{\dashv e}(k)$ (and $P_{\Delta_{\mathrm{S}}}^{\dashv f}(k)$ ) is the steady state probability of the event that a packet of $\mathrm{S}$ is aged for $k$ time slots at $\mathrm{D}$, and at the end of this time Q is empty (full, resp.). Also, $P_{\Omega_{\mathrm{S}}}^{\dashv f}(k)$ is the steady state probability of a packet of $\mathrm{S}$ to wait for $k$ time slots at $\mathrm{Q}$.

The steady state probabilities $C_{\mathrm{S}>\mathrm{R}}^{e}(m), C_{\mathrm{S}>\mathrm{R}}^{f-i o}(m)$ and $C_{\mathrm{S}>\mathrm{R}}^{f-o}(m)$ are found by following the same approach as in the Sec. V-A. To that end, let $T_{\mathrm{S}>\mathrm{R}}^{e}$ refer to the set of all time slots at which $\Delta_{\mathrm{S}}(\ell)>\Delta_{\mathrm{R}}(\ell)$ assuming that $\mathrm{P}_{0}$ has seen $\mathrm{Q}$ in empty state when it is received at $\mathrm{D}$. Also, $T_{\mathrm{S}>\mathrm{R}}^{f-o o}\left(\right.$ and $T_{\mathrm{S}>\mathrm{R}}^{f-i o}$ ) denotes the set of all time slots where $\Delta_{\mathrm{S}}(\ell)>\Delta_{\mathrm{R}}(\ell)$ and $\mathrm{P}_{0}$ finds $\mathrm{P}^{\mathrm{S}}$ (another packet than $\mathrm{P}^{\mathrm{S}}$, resp.) in $\mathrm{Q}$ upon arrival to D. A QBD type Markov chain, namely $\Xi_{\mathrm{S}>\mathrm{R}}^{e}$ (and $\Xi_{\mathrm{S}>\mathrm{R}}^{f}$ ), is developed (based on $\Xi_{R}$ ) to model the age cycle of $P_{0}$, until the moment that another packet of $\mathrm{S}$ or $\mathrm{R}$ is received at $\mathrm{D}$, provided that $\ell \in T_{\mathrm{S}>\mathrm{R}}^{e}\left(\ell \in T_{\mathrm{S}>\mathrm{R}}^{f-o o} \cup T_{\mathrm{S}>\mathrm{R}}^{f-i o}\right.$, resp.). Refer to Fig. 8 for further details.

In the QBD $\Xi_{\mathrm{S}>\mathrm{R}}^{e}$, depicted in Fig. 9, $\mathrm{P}_{0}$ begins its journey from level 1 at phase $\mathrm{E}$, which indicates that $\mathrm{P}_{0}$ has entered $\mathrm{Q}$ when it is empty. Then the level of $\Xi_{\mathrm{S}>\mathrm{R}}^{e}$ increases as $\Delta_{\mathrm{R}}(\ell)$ evolves, until a successful transmission from $\mathrm{R}$ or $\mathrm{S}$ to $\mathrm{D}$. During this time, accepting a new packet of $\mathrm{S}$ in $\mathrm{Q}$ changes the phase of $\Xi_{S>R}^{e}$ to $F$. At the end, once a new packet of R or $\mathrm{S}$ is received at $\mathrm{D}$, the auxiliary phase $\mathrm{T}$ helps to restart $\Xi_{\mathrm{S}>\mathrm{R}}^{e}$ from level 1, such that its QBD form is maintained.

Ignoring the phase $\mathrm{T}$, we can obtain from $\Xi_{\mathrm{S}>\mathrm{R}}^{e}$

$$
\begin{aligned}
\lim _{\ell \rightarrow \infty} \operatorname{Pr}\left(\Delta_{\mathrm{R}}(\ell)=m \mid \ell \in T_{\mathrm{S}>\mathrm{R}}^{e}\right)= \\
\quad \frac{\pi_{\mathrm{S}>\mathrm{R}}^{e}(m, \mathrm{E})+\pi_{\mathrm{S}>\mathrm{R}}^{e}(m, \mathrm{~F})}{\sum_{m=1}^{\infty} \pi_{\mathrm{S}>\mathrm{R}}^{e}(m, \mathrm{E})+\pi_{\mathrm{S}>\mathrm{R}}^{e}(m, \mathrm{~F})}, \quad m \geqslant 1,
\end{aligned}
$$

where $\pi_{\mathrm{S}>\mathrm{R}}^{e}(x)$ is the stationary distribution of $\Xi_{\mathrm{S}>\mathrm{R}}^{e}$ at state $x$ and $\pi_{\mathrm{S}>\mathrm{R}}^{e}(1, \mathrm{~F})=0$. Following the same approach as in (21) and (22), it is straightforward to write

$$
\lim _{\ell \rightarrow \infty} \operatorname{Pr}\left(\Delta_{\mathrm{R}}(\ell)=m \mid \ell \in T_{\mathrm{S}>\mathrm{R}}^{e}\right)=C_{\mathrm{S}>\mathrm{R}}^{e}(m) \frac{q^{e}}{\tau_{\mathrm{S}>\mathrm{R}}^{e}},
$$

where $\tau_{\mathrm{S}>\mathrm{R}}^{e} \triangleq \lim _{\ell \rightarrow \infty} \operatorname{Pr}\left(\ell \in T_{\mathrm{S}>\mathrm{R}}^{e}\right)$ is the steady state probability of being in stage $T_{\mathrm{S}>\mathrm{R}}^{e}$ and is found in Section V-C.

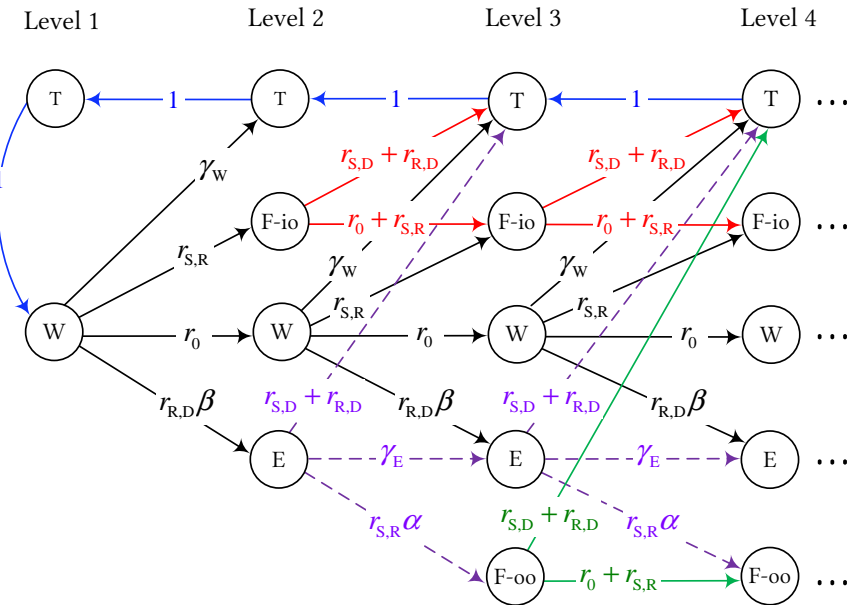

Fig. 10: The transition diagram of $\Xi_{\mathrm{S}>\mathrm{R}}^{f}$, where $\gamma_{\mathrm{W}} \triangleq r_{\mathrm{S}, \mathrm{D}}+r_{\mathrm{R}, \mathrm{D}}(1-\beta)$ and $\gamma_{\mathrm{E}} \triangleq r_{0}+r_{\mathrm{S}, \mathrm{R}}(1-\alpha)$

Likewise, $\Xi_{\mathrm{S}>\mathrm{R}}^{f}$ (see Fig. 10) models the age cycle of $\mathrm{P}_{0}$ when $\mathrm{Q}$ is full upon its arrival to D. However, as described earlier, the existing packet in $\mathrm{Q}$ at time slot $\ell-m$ makes decisive difference in calculating $p(m+k, m)$. Suppose that when $\mathrm{P}_{0}$ starts its journey in $\Xi_{\mathrm{S}>\mathrm{R}}^{f}$ from level 1 at phase $\mathrm{W}$ (conveying that $\mathrm{Q}$ is full when $\mathrm{P}_{0}$ arrives), it finds $\mathrm{P}^{\mathrm{S}}$ in $\mathrm{Q}$. Once $\mathrm{P}^{\mathrm{S}}$ is delivered to $\mathrm{D}$ the phase changes from $\mathrm{W}$ to $\mathrm{E}$. After that, the phase may change from $\mathrm{E}$ to $\mathrm{F}$-oo if another packet of $\mathrm{S}$ is accepted in Q. Thus, when $\Xi_{\mathrm{S}>\mathrm{R}}^{f}$ is at phases $\mathrm{E}$ and $\mathrm{F}-\mathrm{oo}, \mathrm{P}^{\mathrm{S}}$ has been delivered to $\mathrm{D}$ out of order while the phase $\mathrm{W}$ indicates that it has not yet been received at $\mathrm{D}$. Finally, once a new packet of $\mathrm{R}$ or $\mathrm{S}$ is received at $\mathrm{D}$, the auxiliary phase $T$ restarts $\Xi_{S>R}^{f}$. Also, once $\mathrm{P}^{S}$ is replaced, the phase changes from $\mathrm{W}$ to $\mathrm{F}$-io and then to $\mathrm{T}$ in some future time slot. The phase F-io is introduced to account for the cases where $\ell \in T_{\mathrm{S}>\mathrm{R}}^{f-i o}$ and is not reachable to $\backslash$ from $\mathrm{E}$ or F-oo. More specifically, suppose that a packet other than $\mathrm{P}^{\mathrm{S}}$, e.g., $\mathrm{P}$, is in $\mathrm{Q}$ when $\mathrm{P}_{0}$ arrives to $\mathrm{D}$. In this case (i.e., $\left.\ell \in T_{\mathrm{S}>\mathrm{R}}^{f-i o}\right) \Xi_{\mathrm{S}>\mathrm{R}}^{f}$ starts at phase $\mathrm{W}$ in level 1 and evolves as time goes. At any time slot, if $\mathrm{P}^{\prime}$ or another packet of $\mathrm{S}$ is delivered to $\mathrm{D}, \Xi_{\mathrm{S}>\mathrm{R}}^{f}$ is restarted through the phase T. However, if $\mathrm{P}$ ' is replaced, phase of $\Xi_{\mathrm{S}>\mathrm{R}}^{f}$ changes to F-io and remains unchanged until a successful transmission from $\mathrm{S}$ or $\mathrm{R}$ to $\mathrm{D}$. Therefore, we have

$$
\begin{aligned}
& \lim _{\ell \rightarrow \infty} \operatorname{Pr}\left(\Delta_{\mathrm{R}}(\ell)=m \mid \ell \in T_{\mathrm{S}>\mathrm{R}}^{f-o o}\right)= \\
& \frac{\pi_{\mathrm{S}>\mathrm{R}}^{f}(m, \mathrm{E})+\pi_{\mathrm{S}>\mathrm{R}}^{f}(m, \mathrm{~F}-\mathrm{oo})}{\sum_{m=2}^{\infty} \pi_{\mathrm{S}>\mathrm{R}}^{f}(m, \mathrm{E})+\pi_{\mathrm{S}>\mathrm{R}}^{f}(m, \mathrm{~F}-\mathrm{oo})}, \quad m \geqslant 2, \\
& \lim _{\ell \rightarrow \infty} \operatorname{Pr}\left(\Delta_{\mathrm{R}}(\ell)=m \mid \ell \in T_{\mathrm{S}>\mathrm{R}}^{f-o o}\right)= \\
& \frac{\pi_{\mathrm{S}>\mathrm{R}}^{f}(m, \mathrm{~W})+\pi_{\mathrm{S}>\mathrm{R}}^{f}(m, \mathrm{~F} \text {-io })}{\sum_{m=1}^{\infty} \pi_{\mathrm{S}>\mathrm{R}}^{f}(m, \mathrm{~W})+\pi_{\mathrm{S}>\mathrm{R}}^{f}(m, \mathrm{~F}-\mathrm{io})}, \quad m \geqslant 1,
\end{aligned}
$$

where $\pi_{\mathrm{S}>\mathrm{R}}^{f}(x)$ is the stationary distribution of $\Xi_{\mathrm{S}>\mathrm{R}}^{f}$ at state $x$, assuming $\pi_{\mathrm{S}>\mathrm{R}}^{e}(2, \mathrm{~F}-\mathrm{oo})=0$ and $\pi_{\mathrm{S}>\mathrm{R}}^{e}(1, \mathrm{~F}$-io $)=0$. Note that, $\operatorname{Pr}\left(\Delta_{\mathrm{R}}(\ell)=1 \mid \ell \in T_{\mathrm{S}>\mathrm{R}}^{f-o o}\right)=0$ due to the required time slot to deliver $\mathrm{P}^{\mathrm{S}}$ to D. Now, as in (21) and (22), we have

$\lim _{\ell \rightarrow \infty} \operatorname{Pr}\left(\Delta_{\mathrm{R}}(\ell)=m \mid \ell \in T_{\mathrm{S}>\mathrm{R}}^{f-o o}\right)=C_{\mathrm{S}>\mathrm{R}}^{f-o o}(m) \frac{1}{\tau_{\mathrm{S}>\mathrm{R}}^{f-o o}}$, 


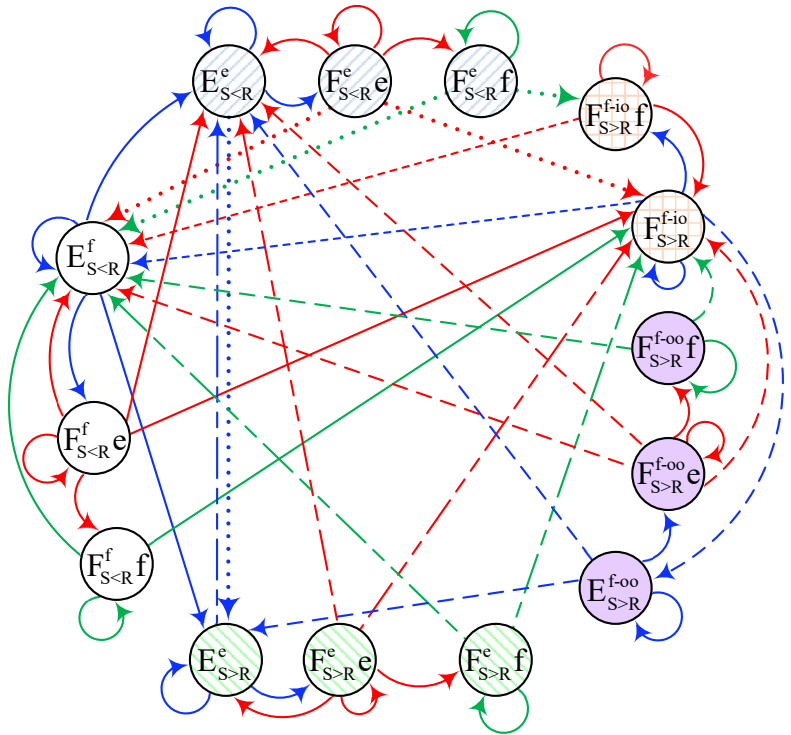

Fig. 11: The transition diagram of $\Xi$

$\lim _{\ell \rightarrow \infty} \operatorname{Pr}\left(\Delta_{\mathrm{R}}(\ell)=m \mid \ell \in T_{\mathrm{S}>\mathrm{R}}^{f-i o}\right)=C_{\mathrm{S}>\mathrm{R}}^{f-i o}(m) \frac{q^{f}}{\tau_{\mathrm{S}>\mathrm{R}}^{f-i o}}$,

in which $\tau_{\mathrm{S}>\mathrm{R}}^{f-o o} \triangleq \lim _{\ell \rightarrow \infty} \operatorname{Pr}\left(\ell \in T_{\mathrm{S}>\mathrm{R}}^{f-o o}\right)$ and $\tau_{\mathrm{S}>\mathrm{R}}^{f-i o} \triangleq \lim _{\ell \rightarrow \infty} \operatorname{Pr}(\ell \in$ $\left.T_{\mathrm{S}>\mathrm{R}}^{f-i o}\right)$ denote the steady state probabilities of being in stages $T_{\mathrm{S}>\mathrm{R}}^{f-o o}$ and $T_{\mathrm{S}>\mathrm{R}}^{f-i o}$, respectively.

\section{Stationary Distribution of Stage of $\Psi(\ell)$}

This section provides the stationary distribution of different stages of $\Psi(\ell)$. To that end, a Markov chain (named as $\Xi$ ) is proposed that models the evolution of $\Psi(\ell)$ between different stages as time goes. States of $\Xi$ are mostly inspired by the phases in the aforementioned QBDs at each stage, namely $\Xi_{\mathrm{S}<\mathrm{R}}^{e}, \Xi_{\mathrm{S}<\mathrm{R}}^{f}, \Xi_{\mathrm{S}>\mathrm{R}}^{e}$ and $\Xi_{\mathrm{S}>\mathrm{R}}^{f}$. Particularly, each state $\Theta_{\sigma}^{\epsilon} \theta$ in $\Xi$ is designated such that the subscript $\sigma$ and superscript $\epsilon$ indicate the corresponding stage of that state, i.e., $\sigma \in\{\mathrm{S}<$ $\mathrm{R}, \mathrm{S}>\mathrm{R}\}$ indicates whether $\Delta_{\mathrm{S}}(\ell)<\Delta_{\mathrm{R}}(\ell)$ or $\Delta_{\mathrm{S}}(\ell)>$ $\Delta_{\mathrm{R}}(\ell)$ at time slot $\ell$, while $\epsilon \in\{\mathrm{e}, \mathrm{f}\}$ shows the state of $\mathrm{Q}$ when the last received packet at $\mathrm{D}$ (from either $\mathrm{S}$ or $\mathrm{R}$ ) is generated. Also, $\Theta \in\{\mathrm{E}, \mathrm{F}\}$ specifies whether $\mathrm{Q}$ is empty or full at the current time slot $\ell$, while $\theta \in\{e, f\}$ determines whether the current packet in $Q$ has been accepted when it was empty or full. Notice that, in state $\mathrm{F}_{\mathrm{S}>\mathrm{R}}^{f-i o}$, it is not necessary to know the state of $\mathrm{Q}$ at the moment that the current packet in $\mathrm{Q}$ has been accepted, because, once this packet is delivered to $\mathrm{D}$, the state changes to $\mathrm{E}_{\mathrm{S}>\mathrm{R}}^{f-o o}$ anyway (refer to transition from $\mathrm{W}$ to $\mathrm{F}-o o$ in $\Xi_{\mathrm{S}>\mathrm{R}}^{f}$ ).

Fig. 11 shows the transition diagram of $\Xi$ and Table III gathers the corresponding transition probabilities. Finding the stationary distribution of $\Xi$, yields the steady state probabilities of being at each stage, i.e., $\tau_{\mathrm{S}<\mathrm{R}}^{e}, \tau_{\mathrm{S}<\mathrm{R}}^{f}, \tau_{\mathrm{S}>\mathrm{R}}^{e}, \tau_{\mathrm{S}>\mathrm{R}}^{f-i o}$ and $\tau_{\mathrm{S}>\mathrm{R}}^{f-o o}$.

\section{Optimal CoOperation Policy}

In this paper, we quantify the simultaneity of the source and relay AoI sequences through the expected value of the SDAoI sequence denoted by $\bar{\Gamma}(\alpha, \beta)$ when the relay node R employs the acceptance and relaying probabilities, $\alpha$ and $\beta$, respectively. Specifically, we seek to minimize $\bar{\Gamma}(\alpha, \beta)$ while the expected value of the steady state PAoI sequence of R, denoted by $\bar{\Lambda}_{R}$, is forced to have a value less than a specific threshold. Mathematically, we address the so-called Constrained Least Mean Squared Difference (CLMSD) optimization problem for which we obtain

$\bar{\Gamma}^{*} \triangleq \min _{0 \leqslant \alpha, \beta \leqslant 1} \bar{\Gamma}(\alpha, \beta) \quad$ such that $\bar{\Lambda}_{\mathrm{R}} \leqslant\left(1+h_{\mathrm{R}}\right) \bar{\Lambda}_{\mathrm{R}}^{\mathrm{NC}}$,

where $\bar{\Lambda}_{\mathrm{R}}^{\mathrm{NC}}$ denotes $\bar{\Lambda}_{\mathrm{R}}$ in the non-cooperative network, i.e., $\alpha=0$ or $\beta=0$, and the non-negative parameter $h_{\mathrm{R}}$ determines the maximum allowed increase in $\bar{\Lambda}_{\mathrm{R}}$ with respect to $\bar{\Lambda}_{\mathrm{R}}^{\mathrm{NC}}$. Note that, in the non-cooperative network, $\mathrm{R}$ does not devote any resources to relay the packets of $\mathrm{S}$ and therefore $\bar{\Lambda}_{\mathrm{R}}$ is minimized in this case. In order to obtain the CLMSD policy in (35), called the optimal cooperation policy hereafter, we firstly derive $\bar{\Lambda}_{\mathrm{R}}$ in closed form in Lemma 3 and subsequently, we reduce the optimization problem (35) to a non-constrained optimization problem but over a specific feasible region for the parameters $\alpha$ and $\beta$ for which the constraint is a-priori known to be satisfied.

Lemma 3: The average PAoI for the packets of $\mathrm{R}$ is $\bar{\Lambda}_{\mathrm{R}}=$ $1+1 / \bar{\lambda}_{R}$ where $\bar{\lambda}_{R}$ is the average rate of departure of the packets of $\mathrm{R}$ and equals

$$
\bar{\lambda}_{\mathrm{R}}=\frac{\left(r_{\mathrm{S}, \mathrm{D}}+r_{\mathrm{R}, \mathrm{D}} \beta\right) r_{\mathrm{R}, \mathrm{D}}+r_{\mathrm{S}, \mathrm{R}} \alpha r_{\mathrm{R}, \mathrm{D}}(1-\beta)}{r_{\mathrm{S}, \mathrm{D}}+r_{\mathrm{R}, \mathrm{D}} \beta+r_{\mathrm{S}, \mathrm{R}} \alpha} .
$$

Proof: Note that the peak age corresponding to each packet of $\mathrm{R}$ consists of two parts: the transmission time of the packet which is one time slot, and the time till the arrival of the next packet of $\mathrm{R}$ to $\mathrm{D}$. Thus, $\bar{\Lambda}_{\mathrm{R}}=1+E\left[d_{\mathrm{R}}\right]$ in which $E\left[d_{\mathrm{R}}\right]$ is the average inter-departure time of the packets of $R$. To find $E\left[d_{\mathrm{R}}\right]$, consider a queuing system composed of the server of $\mathrm{R}$ where a packet of $\mathrm{R}$ is generated and ready to send at each time slot. By using Little's law [33], $E\left[d_{\mathrm{R}}\right]=1 / \bar{\lambda}_{\mathrm{R}}$ in which $\bar{\lambda}_{\mathrm{R}}$ is the average departure rate of packets from the queuing system and can be written as $\bar{\lambda}_{\mathrm{R}}=r_{\mathrm{R}, \mathrm{D}} q^{e}+r_{\mathrm{R}, \mathrm{D}}(1-\beta) q^{f}$, where $q^{e}$ and $q^{f}$ are given by (23). The lemma is concluded by substituting the expressions for $q^{e}$ and $q^{f}$.

Having $\bar{\Lambda}_{\mathrm{R}}=1+1 / \bar{\lambda}_{\mathrm{R}}$, the inequality constraint in (35) can be rewritten in the form $\bar{\lambda}_{\mathrm{R}} \geqslant \lambda_{0}$ where $\lambda_{0} \triangleq$ $1 /\left(\left(1+h_{\mathrm{R}}\right) \bar{\Lambda}_{\mathrm{R}}^{\mathrm{NC}}-1\right)$. Now, by applying (36) and after some manipulations, this constraint is reduced to

$$
\left\{\begin{array}{l}
\alpha \leqslant \alpha_{0} ; \quad \beta_{0}<\beta \leqslant 1, \\
\alpha \geqslant \alpha_{0} ; \quad 0 \leqslant \beta \leqslant \beta_{0} .
\end{array}\right.
$$

in which

$$
\alpha_{0} \triangleq \frac{r_{\mathrm{R}, \mathrm{D}} \beta_{0}}{r_{\mathrm{S}, \mathrm{R}}} \frac{\beta+r_{\mathrm{S}, \mathrm{D}} / r_{\mathrm{R}, \mathrm{D}}}{\beta-\beta_{0}}, \quad \beta_{0} \triangleq 1-\frac{\lambda_{0}}{r_{\mathrm{R}, \mathrm{D}}} .
$$

Note that in the non-cooperative network we have $\alpha=\beta=0$, thus, $\bar{\Lambda}_{\mathrm{R}}^{\mathrm{NC}}=1 / r_{\mathrm{R}, \mathrm{D}}$ is a corollary of Lemma 3 . Henceforth, it is straightforward to see that $\beta_{0} \geqslant 0$. Accordingly, $\beta<\beta_{0}$ results in $\alpha_{0}<0$ (see (38)), which concludes that the constraint on $\alpha$ in the second line of (37) is always satisfied. However, since $\alpha_{0}>0$ for $\beta>\beta_{0}$, the constraint in the first line of (37) becomes $\alpha \leqslant \min \left(\alpha_{0}, 1\right)$ for $\beta_{0}<\beta \leqslant 1$. Therefore, (35) reduces to minimizing $\bar{\Gamma}(\alpha, \beta)$ unconstrainedly over the feasible region of the pair $(\alpha, \beta)$, that is, $0 \leqslant \alpha \leqslant 1$ for 
TABLE III: Transition probabilities in $\Xi$

\begin{tabular}{|c|c|c|c|c|c|c|c|c|c|c|c|c|c|c|}
\hline & $\mathrm{E}_{\mathrm{S}<\mathrm{R}}^{e}$ & $\mathrm{~F}_{\mathrm{S}<\mathrm{R}}^{e}$ & $\mathrm{~F}_{\mathrm{S}<\mathrm{R}}^{e} f$ & $\mathrm{E}_{\mathrm{S}<\mathrm{R}}^{f}$ & $\mathrm{~F}_{\mathrm{S}<\mathrm{R}}^{f} e$ & $\mathrm{~F}_{\mathrm{S}<\mathrm{R}}^{f} f$ & $\mathrm{E}_{\mathrm{S}>\mathrm{R}}^{e}$ & $\mathrm{~F}_{\mathrm{S}>\mathrm{R}}^{e}$ & $\mathrm{~F}_{\mathrm{S}>\mathrm{R}}^{e} f$ & $\mathrm{~F}_{\mathrm{S}>\mathrm{R}}^{f-i o}$ & $\mathrm{~F}_{\mathrm{S}>\mathrm{R}}^{f-i o} f$ & $\mathrm{E}_{\mathrm{S}>\mathrm{R}}^{f-o o}$ & $\mathrm{~F}_{\mathrm{S}>\mathrm{R}}^{f-o o} e$ & $\mathrm{~F}_{\mathrm{S}>\mathrm{R}}^{f-o o} f$ \\
\hline $\mathrm{E}_{\mathrm{S}<\mathrm{R}}^{e}$ & $\gamma_{1}^{(a)}$ & $r_{\mathrm{S}, \mathrm{R}} \alpha$ & 0 & 0 & 0 & 0 & $r_{\mathrm{R}, \mathrm{D}}$ & 0 & 0 & 0 & 0 & 0 & 0 & 0 \\
\hline $\mathrm{F}_{\mathrm{S}<\mathrm{R}}^{e}$ & $r_{\mathrm{R}, \mathrm{D}} \beta$ & $r_{0}$ & $r_{\mathrm{S}, \mathrm{R}}$ & $r_{\mathrm{S}, \mathrm{D}}$ & 0 & 0 & 0 & 0 & 0 & $r_{\mathrm{R}, \mathrm{D}}(1-\beta)$ & 0 & 0 & 0 & 0 \\
\hline $\mathrm{F}_{\mathrm{S}<\mathrm{R}}^{e} f$ & 0 & 0 & $r_{0}+r_{\mathrm{S}, \mathrm{R}}$ & $\gamma_{2}^{(b)}$ & 0 & 0 & 0 & 0 & 0 & $r_{\mathrm{R}, \mathrm{D}}(1-\beta)$ & 0 & 0 & 0 & 0 \\
\hline $\mathrm{E}_{\mathrm{S}<\mathrm{R}}^{f}$ & $r_{\mathrm{S}, \mathrm{D}}$ & 0 & 0 & $\gamma_{3}^{(\mathrm{c})}$ & $r_{\mathrm{S}, \mathrm{R}} \alpha$ & 0 & $r_{\mathrm{R}, \mathrm{D}}$ & 0 & 0 & 0 & 0 & 0 & 0 & 0 \\
\hline $\mathrm{F}_{\mathrm{S}<\mathrm{R}}^{f} e$ & $r_{\mathrm{R}, \mathrm{D}} \beta$ & 0 & 0 & $r_{\mathrm{S}, \mathrm{D}}$ & $r_{0}$ & $r_{\mathrm{S}, \mathrm{R}}$ & 0 & 0 & 0 & $r_{\mathrm{R}, \mathrm{D}}(1-\beta)$ & 0 & 0 & 0 & 0 \\
\hline $\mathrm{F}_{\mathrm{S}<\mathrm{R}}^{f} f$ & 0 & 0 & 0 & $\gamma_{2}$ & 0 & $r_{0}+r_{\mathrm{S}, \mathrm{R}}$ & 0 & 0 & 0 & $r_{\mathrm{R}, \mathrm{D}}(1-\beta)$ & 0 & 0 & 0 & 0 \\
\hline $\mathrm{E}_{\mathrm{S}>\mathrm{R}}^{e}$ & $r_{\mathrm{S}, \mathrm{D}}$ & 0 & 0 & 0 & 0 & 0 & $\gamma_{4}^{(\mathrm{d})}$ & $r_{\mathrm{S}, \mathrm{R}} \alpha$ & 0 & 0 & 0 & 0 & 0 & 0 \\
\hline $\mathrm{F}_{\mathrm{S}>\mathrm{R}}^{e}$ & $r_{\mathrm{R}, \mathrm{D}} \beta$ & 0 & 0 & $r_{\mathrm{S}, \mathrm{D}}$ & 0 & 0 & 0 & $r_{0}$ & $r_{\mathrm{S}, \mathrm{R}}$ & $r_{\mathrm{R}, \mathrm{D}}(1-\beta)$ & 0 & 0 & 0 & 0 \\
\hline $\mathrm{F}_{\mathrm{S}>\mathrm{R}}^{e} f$ & 0 & 0 & 0 & $\gamma_{2}$ & 0 & 0 & 0 & 0 & $r_{0}+r_{\mathrm{S}, \mathrm{R}}$ & $r_{\mathrm{R}, \mathrm{D}}(1-\beta)$ & 0 & 0 & 0 & 0 \\
\hline $\mathrm{F}_{\mathrm{S}>\mathrm{R}}^{f-i o}$ & 0 & 0 & 0 & $r_{\mathrm{S}, \mathrm{D}}$ & 0 & 0 & 0 & 0 & 0 & $\gamma_{5}^{(\mathrm{e})}$ & $r_{\mathrm{S}, \mathrm{R}}$ & $r_{\mathrm{R}, \mathrm{D}} \beta$ & 0 & 0 \\
\hline $\mathrm{F}_{\mathrm{S}>\mathrm{R}}^{f-i o} f$ & 0 & 0 & 0 & $\gamma_{2}$ & 0 & 0 & 0 & 0 & 0 & $r_{\mathrm{R}, \mathrm{D}}(1-\beta)$ & $r_{0}+r_{\mathrm{S}, \mathrm{R}}$ & 0 & 0 & 0 \\
\hline $\mathrm{E}_{\mathrm{S}>\mathrm{R}}^{f-o o}$ & $r_{\mathrm{S}, \mathrm{D}}$ & 0 & 0 & 0 & 0 & 0 & $r_{\mathrm{R}, \mathrm{D}}$ & 0 & 0 & 0 & 0 & $\gamma_{3}$ & $r_{\mathrm{S}, \mathrm{R}} \alpha$ & 0 \\
\hline $\mathrm{F}_{\mathrm{S}>\mathrm{R}}^{f-o o} e$ & $r_{\mathrm{R}, \mathrm{D}} \beta$ & 0 & 0 & $r_{\mathrm{S}, \mathrm{D}}$ & 0 & 0 & 0 & 0 & 0 & $r_{\mathrm{R}, \mathrm{D}}(1-\beta)$ & 0 & 0 & $r_{0}$ & $r_{\mathrm{S}, \mathrm{R}}$ \\
\hline $\mathrm{F}_{\mathrm{S}>\mathrm{R}}^{f-o o} f$ & 0 & 0 & 0 & $\gamma_{2}$ & 0 & 0 & 0 & 0 & 0 & $r_{\mathrm{R}, \mathrm{D}}(1-\beta)$ & 0 & 0 & 0 & $r_{0}+r_{\mathrm{S}, \mathrm{R}}$ \\
\hline
\end{tabular}

(a) $\gamma_{1} \triangleq r_{0}+r_{\mathrm{S}, \mathrm{R}}(1-\alpha)+r_{\mathrm{S}, \mathrm{D}} \quad$ (b) $\gamma_{2} \triangleq r_{\mathrm{S}, \mathrm{D}}+r_{\mathrm{R}, \mathrm{D}} \beta$

(d) $\gamma_{4} \triangleq r_{0}+r_{\mathrm{S}, \mathrm{R}}(1-\alpha)+r_{\mathrm{R}, \mathrm{D}}$

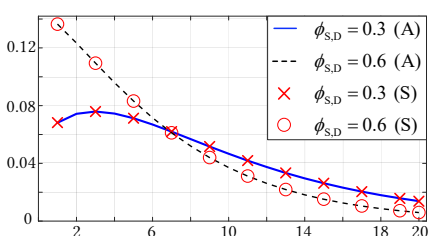

(a) $p_{\Delta_{\mathrm{S}}}(n)$

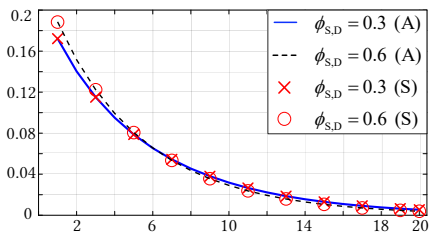

(c) $p_{\Lambda_{\mathrm{S}}}(n)$

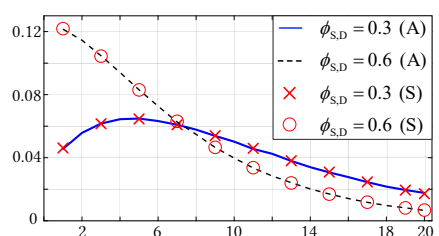

(b) $p_{\Delta_{\mathrm{R}}}(n)$

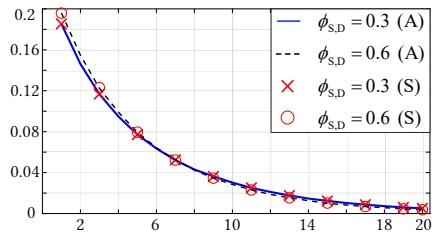

(d) $p_{\Lambda_{\mathrm{R}}}(n)$
Fig. 12: The steady state pmf of the source and relay AoI and PAoI sequences for two values of $\phi_{\mathrm{S}, \mathrm{D}}$ when $\phi_{\mathrm{R}, \mathrm{D}}=0.9, \phi_{\mathrm{S}, \mathrm{R}}=1$ and $\alpha=\beta=0.5$.

$0 \leqslant \beta \leqslant \beta_{0}$ and $0 \leqslant \alpha \leqslant \min \left(\alpha_{0}, 1\right)$ for $\beta_{0}<\beta \leqslant 1$. As illustrated later by numerical results, the optimal cooperation policy improves the performance of network significantly in comparison with the no-cooperation and full-cooperation policies, moreover there are situations in which it slightly outperforms the optimal pure-replacement policy.

\section{NumericAl Results}

In this section, marginal and joint pmfs of the AoI and PAoI sequences of $\mathrm{S}$ and $\mathrm{R}$ are numerically obtained using the proposed analytical model, and the results are confirmed by numerous simulations. Subsequently, we use exhaustive search to find $\bar{\Gamma}^{*}$ and compare the CMLSD policy with nocooperation policy, i.e., $\alpha=0$ or $\beta=0$, full-cooperation policy, i.e., $\alpha=\beta=1$, where $\mathrm{R}$ accepts all the incoming packets from $\mathrm{S}$ and prioritizes them over its own packets when making transmission decisions, and optimal pure-replacement policy, i.e., optimization over the parameter $\beta$ is sought while $\alpha$ is fixed to unity, that is, where all the incoming packets are accepted at $\mathrm{Q}$ but relayed optimally such that $\bar{\Gamma}(1, \beta)$ is (c) $\gamma_{3} \triangleq r_{0}+r_{\mathrm{S}, \mathrm{R}}(1-\alpha)$

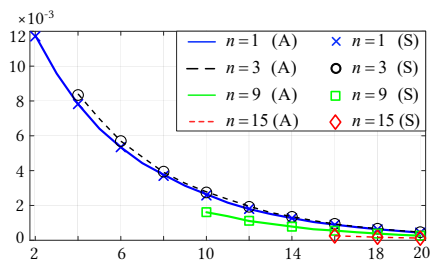

(a) $p(n, m)$ vs $n$, for $m<n$

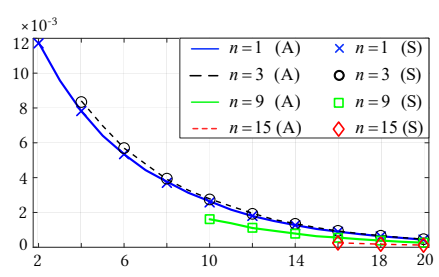

(b) $p(n, m)$ vs $m$, for $n<m$
Fig. 13: The steady state joint pmf of the source and relay AoI sequences for $\phi_{\mathrm{S}, \mathrm{D}}=0.3$ when $\phi_{\mathrm{R}, \mathrm{D}}=0.9, \phi_{\mathrm{S}, \mathrm{R}}=1$ and $\alpha=\beta=0.5$.

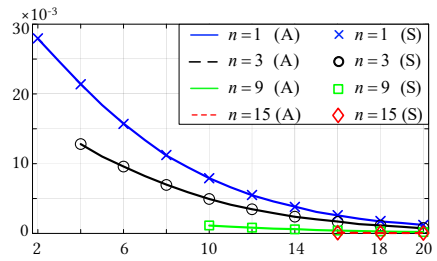

(a) $p(n, m)$ vs $n$, for $m<n$

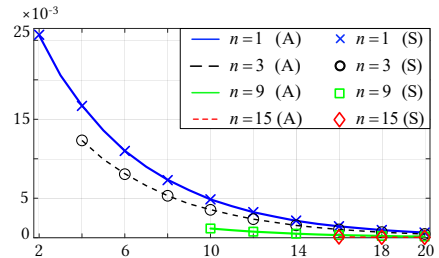

(b) $p(n, m)$ vs $m$, for $n<m$
Fig. 14: The steady state joint pmf of the source and relay AoI sequences for $\phi_{\mathrm{S}, \mathrm{D}}=0.6$ when $\phi_{\mathrm{R}, \mathrm{D}}=0.9, \phi_{\mathrm{S}, \mathrm{R}}=1$ and $\alpha=\beta=0.5$.

minimized. In the studied scenarios, we fix $p_{\mathrm{S}}=p_{\mathrm{R}}=0.65$, but the impact of the system parameters $\phi_{\mathrm{S}, \mathrm{D}}, \phi_{\mathrm{R}, \mathrm{D}}$, and $\phi_{\mathrm{S}, \mathrm{R}}$ are investigated thoroughly.

\section{A. Model Validation Using Simulations}

In this subsection, we verify our proposed analytical model using simulations. Figs. $12 \mathrm{a}$ and $12 \mathrm{~b}$ depict the marginal pmf of AoI for both nodes for two different values of $\phi_{\mathrm{S}, \mathrm{D}}$ while fixing $\phi_{\mathrm{R}, \mathrm{D}}=0.9, \phi_{\mathrm{S}, \mathrm{R}}=1$ and $\alpha=\beta=0.5$. Also, the pmf of PAoI of nodes and the joint pmf of AoI sequences are depicted in Figs. 12c, 12d and Figs. 13 and 14, respectively. As seen, the obtained results using the proposed analytical model match perfectly well with the simulation results, which are indicated by $\mathrm{A}$ and $\mathrm{S}$ in the legends, respectively.

\section{B. Age-Optimal Cooperation Policy}

Solving the optimization problem in (35), we have found the optimal cooperation policy in various scenarios. More 


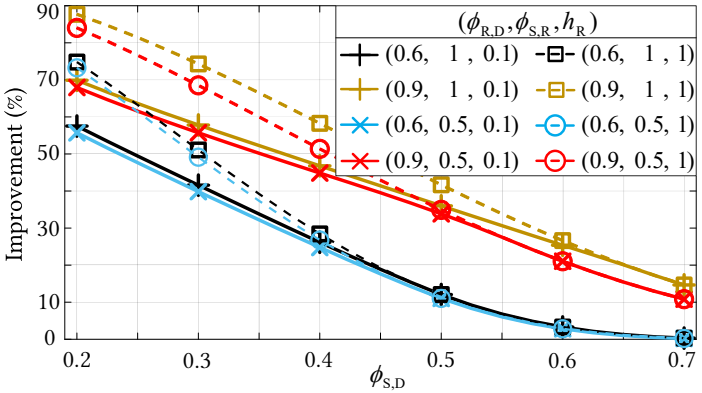

Fig. 15: Percentage of improvement in MSD gained by using the optimal policy with respect to the no-cooperation policy.

specifically, we have considered good and mediocre R-D channel states (i.e., $\phi_{\mathrm{R}, \mathrm{D}}=0.9$ and 0.6 , resp.) and also perfect and medium states for the S-R channel (i.e., $\phi_{\mathrm{S}, \mathrm{R}}=1$ and 0.5 , resp.) while $\phi_{\mathrm{S}, \mathrm{D}}$ changes from 0.2 to 0.7 . Also, the results are obtained where $\bar{\Lambda}_{\mathrm{R}}$ is tightly and loosely constrained (i.e., $h_{\mathrm{R}}=0.1$ and 1 , resp.).

Fig. 15 compares $\bar{\Gamma}$ in a network powered by the optimal policy and the non-cooperative network. As observed, MSD is significantly decreased when the optimal policy is applied which is due to cooperation as well as choosing the optimal policy. The improvement can be up to $90 \%$ especially in the network scenarios with bad S-D channel state, in our numerical examples. As illustrated in Fig. 16, the optimal policy also outperforms the full-cooperation policy for more than 50\% in bad S-D channels. Figs. 15 and 16 reveal that not only cooperation helps to improve MSD, but also the optimal choice of $\alpha$ and $\beta$ can reduce it substantially. In fact, in the full-cooperation policy the AoI of $\mathrm{S}$ is minimized, however, packets of $\mathrm{R}$ have the least priority and experience the longest inter-departure time. On the other hand, in the noncooperative network, $\mathrm{R}$ devotes all of its resources to send its own packets, hence, AoI of $\mathrm{S}$ is maximized unlike AoI of $\mathrm{R}$. However, in the optimal policy, $\mathrm{R}$ adjusts $\alpha$ and $\beta$ such that AoI of the nodes is closest to each other in the way that $\bar{\Gamma}$ is minimized. From a different point of view, devoting time to relay all the incoming packets of $\mathrm{S}$ in the full-cooperative network increases $\bar{\Lambda}_{\mathrm{R}}$ such that in some cases it exceeds the desired threshold, particularly when it is tightly constrained. Fig. 17 compares $\bar{\Lambda}_{\mathrm{R}}^{\mathrm{FC}}\left(\bar{\Lambda}_{\mathrm{R}}\right.$ in the full-cooperative network) with the threshold $\left(1+h_{\mathrm{R}}\right) \bar{\Lambda}_{\mathrm{R}}^{\mathrm{NC}}$. The green and red arrows on this figure indicate the cases where $\bar{\Lambda}_{\mathrm{R}}^{\mathrm{FC}}<\left(1+h_{\mathrm{R}}\right) \bar{\Lambda}_{\mathrm{R}}^{\mathrm{NC}}$ and $\bar{\Lambda}_{\mathrm{R}}^{\mathrm{FC}}>\left(1+h_{\mathrm{R}}\right) \bar{\Lambda}_{\mathrm{R}}^{\mathrm{NC}}$, respectively. As observed, in almost all of the studied tightly-constrained scenarios of this paper, full-cooperation policy leads to undesirable $\bar{\Lambda}_{\mathrm{R}}$.

Fig. 18 compares the optimal policy with the optimal purereplacement policy. As deduced from this figure, optimizing the relaying probability when all packets of $\mathrm{S}$ are accepted at $\mathrm{Q}(\alpha=1)$, is not generally optimal. More specifically, there are some scenarios in which applying a probabilistic admission control on the incoming packets of $\mathrm{S}$, in addition to the relaying prioritization, can decrease MSD even more. As in our numerical examples, in such cases, $\bar{\Lambda}_{\mathrm{R}}$ is tightly constrained and up to $15 \%$ improvement in MSD can be achieved. However, in the majority of the cases, the purereplacement policy results in close to optimal performance.

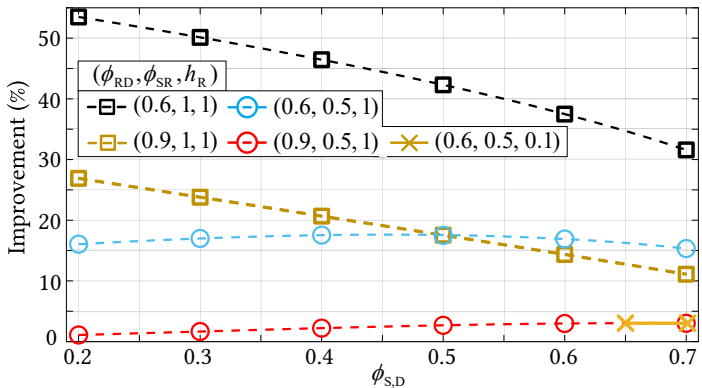

Fig. 16: Percentage of improvement in MSD gained by using the optimal policy with respect to the full-cooperation policy.

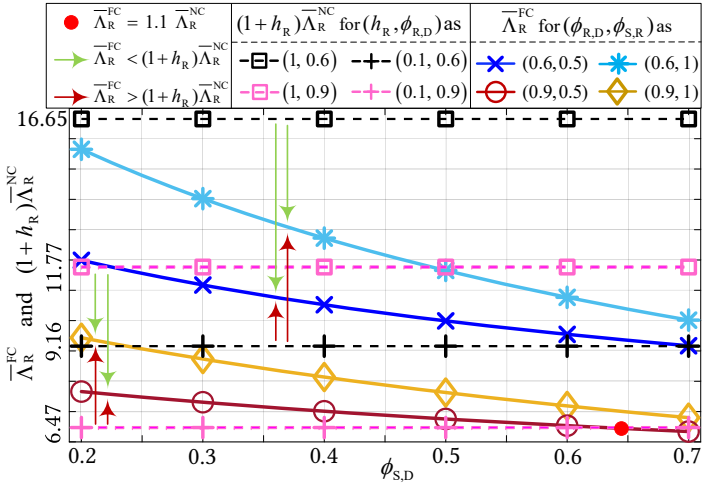

Fig. 17: A comparison between $\bar{\Lambda}_{\mathrm{R}}^{\mathrm{FC}}$ and the threshold $\left(1+h_{\mathrm{R}}\right) \bar{\Lambda}_{\mathrm{R}}^{\mathrm{NC}}$

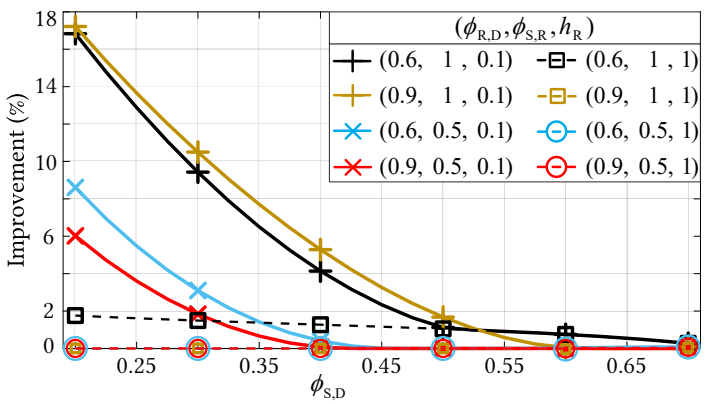

Fig. 18: Percentage of improvement in MSD gained by using the optimal policy with respect to the optimal pure-replacement policy.

\section{CONCLUSION}

In this paper, we investigate a slotted Aloha-based cooperative status update network involving a source and a relay node. Exact marginal pmfs of AoI and also PAoI sequences of both nodes are obtained numerically using QBD-type Markov chain-based stochastic models. Extending this approach, we also find the joint pmf of the AoI sequences of the two nodes. Subsequently, we obtain the optimal cooperation policy in order to minimize the MSD of the two sequences subject to constrained average PAoI of the relay node. Our proposed analytical approach to find marginal and joint distributions of AoI and PAoI sequences is first verified by simulations. Moreover, we study MSD for various cooperation policies. As illustrated, the optimal cooperation policy results in substantial improvement in MSD compared to full-cooperation and nocooperation policies especially when the success probability of the S-D link is low. We also show that the optimal purereplacement policy is not generally optimal, however, the improvements of the optimal policy over it are not as significant. 


\section{REFERENCES}

[1] S. Kaul, R. Yates, and M. Gruteser, "Real-time status: How often should one update?" in Proc. IEEE INFOCOM, 2012, pp. 2731-2735.

[2] M. Costa, M. Codreanu, and A. Ephremides, "Age of information with packet management," in IEEE ISIT, 2014, pp. 1583-1587.

[3] R. D. Yates, Y. Sun, D. R. Brown, S. K. Kaul, E. Modiano, and S. Ulukus, "Age of information: An introduction and survey," IEEE JSAC, vol. 39, no. 5, pp. 1183-1210, 2021.

[4] A. Kosta, N. Pappas, and V. Angelakis, Age of Information: A New Concept, Metric, and Tool. Foundations and Trends ${ }^{\circledR}$ in Networking, 2017, vol. 12, no. 3 .

[5] K. Vaezi and F. Ashtiani, "Delay-optimal cooperation policy in a slotted Aloha full-duplex wireless network: Static approach," IEEE Syst. Journal, vol. 14, no. 2, pp. 2257-2268, 2020.

[6] B. Li, H. Chen, Y. Zhou, and Y. Li, "Age-oriented opportunistic relaying in cooperative status update systems with stochastic arrivals," in IEEE Conf. GLOBECOM, 2020, pp. 1-6.

[7] M. Moradian and A. Dadlani, "Age of information in scheduled wireless relay networks," in IEEE Conf. WCNC, 2020, pp. 1-6.

[8] K. Vaezi and F. Ashtiani, "Delay-optimal static relaying policy in a slotted Aloha wireless network," in Iran Workshop Commun. Inf. Theory (IWCIT), 2018, pp. 1-6.

[9] S. Kaul, M. Gruteser, V. Rai, and J. Kenney, "Minimizing age of information in vehicular networks," in 8th Ann. IEEE Commun. Society Conf. Sensor, Mesh and Ad Hoc Commun. Netw., 2011, pp. 350-358.

[10] M. Costa, M. Codreanu, and A. Ephremides, "On the age of information in status update systems with packet management," IEEE Trans. Inf. Theory, vol. 62, no. 4, pp. 1897-1910, 2016.

[11] Y. Inoue, H. Masuyama, T. Takine, and T. Tanaka, "A general formula for the stationary distribution of the age of information and its application to single-server queues," IEEE Trans. Inf. Theory, vol. 65, no. 12, pp. $8305-8324,2019$.

[12] N. Akar, O. Doğan, and E. U. Atay, "Finding the exact distribution of (peak) age of information for queues of $\mathrm{PH} / \mathrm{PH} / 1 / 1$ and $\mathrm{M} / \mathrm{PH} / 1 / 2$ type," IEEE Trans. Commun., vol. 68, no. 9, pp. 5661-5672, 2020.

[13] R. D. Yates and S. K. Kaul, "The age of information: Real-time status updating by multiple sources," IEEE Trans. Inf. Theory, vol. 65, no. 3, pp. 1807-1827, 2019.

[14] L. Huang and E. Modiano, "Optimizing age-of-information in a multiclass queueing system," in IEEE ISIT, 2015, pp. 1681-1685.

[15] M. Moltafet, M. Leinonen, and M. Codreanu, "Average age of information for a multi-source $\mathrm{M} / \mathrm{M} / 1$ queueing model with packet management," in IEEE ISIT, 2020, pp. 1765-1769.

[16] V. Tripathi, R. Talak, and E. H. Modiano, "Age of information for discrete time queues," CoRR, vol. abs/1901.10463, 2019. [Online]. Available: http://arxiv.org/abs/1901.10463

[17] A. Kosta, N. Pappas, A. Ephremides, and V. Angelakis, "The age of information in a discrete time queue: Stationary distribution and nonlinear age mean analysis," IEEE JSAC, vol. 39, no. 5, pp. 1352-1364, 2021.

[18] N. Akar and O. Doğan, "Discrete-time queueing model of age of information with multiple information sources," IEEE Internet of Things Journal, 2021.

[19] A. M. Bedewy, Y. Sun, and N. B. Shroff, "Age-optimal information updates in multihop networks," in IEEE ISIT, 2017, pp. 576-580.

[20] B. Li, Q. Wang, H. Chen, Y. Zhou, and Y. Li, "Optimizing information freshness for cooperative IoT systems with stochastic arrivals," IEEE Internet of Things Journal, 2021.

[21] O. Vikhrova, F. Chiariotti, B. Soret, G. Araniti, A. Molinaro, and P. Popovski, "Age of information in multi-hop networks with priorities," in IEEE Conf. GLOBECOM, 2020, pp. 1-6.

[22] D. C. Atabay, E. Uysal, and O. Kaya, "Improving age of information in random access channels," in IEEE Conf. INFOCOM WKSHPS, 2020, pp. 912-917.

[23] A. Munari, "Modern random access: An age of information perspective on irregular repetition slotted Aloha," IEEE Trans. Commun., vol. 69, no. 6, pp. 3572-3585, 2021.

[24] X. Chen, K. Gatsis, H. Hassani, and S. S. Bidokhti, "Age of information in random access channels," in IEEE ISIT, 2020, pp. 1770-1775.

[25] O. T. Yavascan and E. Uysal, "Analysis of slotted Aloha with an age threshold," IEEE JSAC, vol. 39, no. 5, pp. 1456-1470, 2021.

[26] H. Chen, Y. Gu, and S.-C. Liew, "Age-of-information dependent random access for massive IoT networks," in IEEE Conf. INFOCOM WKSHPS, 2020, pp. 930-935.
[27] I. Kadota and E. Modiano, "Age of information in random access networks with stochastic arrivals," in IEEE Conf. INFOCOM, 2021, pp. $1-10$.

[28] A. Baiocchi and I. Turcanu, "Age of information of one-hop broadcast communications in a CSMA network," IEEE Commun. Letters, vol. 25 , no. 1, pp. 294-298, 2021.

[29] A. Maatouk, M. Assaad, and A. Ephremides, "On the age of information in a CSMA environment," IEEE/ACM Trans. Netw., vol. 28, no. 2, pp. 818-831, 2020.

[30] Z. Jiang, S. Zhou, and Z. Niu, "Distributed policy learning based random access for diversified QoS requirements," in IEEE Conf. ICC, 2019, pp. $1-6$.

[31] M. F. Neuts, Matrix-Geometric Solutions in Stochastic Models - An Algorithmic Approach. Dover Publications, 1994.

[32] N. Akar, N. C. Oğuz, and K. Sohraby, "A novel computational method for solving finite QBD processes," Commun. Statist.-Stochastic Models, vol. 16, no. 2, pp. 273-311, 2000.

[33] D. Bertsekas and R. Gallager, Data Networks, ser. 2nd ed. Prentice-Hall Inc., 1992. 